\title{
Recipes for stellar jets: results of combined optical/infrared diagnostics ${ }^{\star}$
}

\author{
L. Podio ${ }^{1}$, F. Bacciotti ${ }^{2}$, B. Nisini ${ }^{3}$, J. Eislöffel ${ }^{4}$, F. Massi ${ }^{2}$, T. Giannini ${ }^{3}$, and T. P. Ray ${ }^{5}$ \\ 1 Dipartimento di Astronomia e Scienza dello Spazio, Universitá degli Studi di Firenze, Largo E. Fermi 2, 50125 Firenze, Italy \\ e-mail: lindapod@arcetri.astro.it \\ 2 INAF-Osservatorio Astrofisico di Arcetri, Largo E. Fermi 5, 50125 Florence, Italy \\ 3 INAF-Osservatorio Astronomico di Roma, via di Frascati 33, 00040 Monte Porzio Catone, Italy \\ 4 Thüringer Landessternwarte Tautenburg, Sternwarte 5, 07778 Tautenburg, Germany \\ 5 School of Cosmic Physics, Dublin Institute for Advanced Studies, 5 Merrion Square, Dublin 2, Ireland
}

Received 5 September 2005 / Accepted 26 May 2006

\section{ABSTRACT}

\begin{abstract}
We examine the conditions of the plasma along a sample of "classical" Herbig-Haro $(\mathrm{HH})$ jets located in the Orion and Vela star forming regions, through combined optical-infrared spectral diagnostics. Our sample includes HH 111, HH 34, HH 83, HH 73, HH 24 C/E, HH 24 J, observed quasi-simultaneously and in the same manner at moderate spatial/spectral resolution. Once intercalibrated, the obtained spectra cover a wide wavelength range from 0.6-2.5 $\mu \mathrm{m}$, including many transitions from regions of different excitation conditions. This allows us to probe the density and temperature stratification which characterises the cooling zones behind the shock fronts along the jet. From the line ratios we derive the variation of the visual extinction along the flow, the electron density and temperature $\left(n_{\mathrm{e}}\right.$ and $T_{\mathrm{e}}$ ), the hydrogen ionisation fraction $x_{\mathrm{e}}$, and the total density $n_{\mathrm{H}}$ in the emission region of different lines. The knowledge of such parameters is essential for testing existing jet models and for planning follow-up high-angular resolution observations.

From the diagnostics of optical forbidden lines we find, on average, that in the examined jets, in the region of optical emission, $n_{\mathrm{e}}$ varies between $50 \mathrm{~cm}^{-3}$ and $3 \times 10^{3} \mathrm{~cm}^{-3}, x_{\mathrm{e}}$ ranges between 0.03 and 0.6 , and the electron temperature $T_{\mathrm{e}}$ is $\sim 1.3 \times 10^{4} \mathrm{~K}$ in the $\mathrm{HH} 111$ and $\mathrm{HH} 34$ jets, while it appears to be higher $\left(1.8 \times 10^{4} \mathrm{~K}\right.$ on average $)$ in the other examined jets. The electron density and temperature derived from [Fe II] lines, turn out to be, respectively, higher and lower in comparison to those determined from optical lines, in agreement with the fact that the [Fe II] lines arise in the more compressed gas located further from the shock front. An even denser component in the jets, with values of $n_{\mathrm{e}}$ up to $10^{6} \mathrm{~cm}^{-3}$ is detected using the ratio of calcium lines.

The derived physical parameters are used to estimate the depletion onto dust grains of calcium and iron with respect to solar abundances. This turns out to be quite substantial, being between $70 \%$ and $0 \%$ for $\mathrm{Ca}$ and $\sim 90 \%$ for Fe. This leads us to suggest that the weak shocks present in the beams are not capable of completely destroying the ambient dust grains, confirming previous theoretical studies. We then derive the mass flux rates, $\dot{M}_{\text {jet }}$, in the flows using two independent methods. Taking into account the filling factor of the emitting gas, $\dot{M}_{\text {jet }}$ is on average $5 \times 10^{-8} M_{\odot} \mathrm{yr}^{-1}$. The associated linear momentum fluxes $\left(\dot{P}_{\text {jet }}=v_{\text {jet }} \dot{M}_{\text {jet }}\right)$ are higher than, or of the same order as, those measured in the coaxial molecular flows, where present, suggesting that the flows are jet driven.

Finally, we discuss differences between jets in our sample. In general, we find that higher ionisation and electron temperatures are associated with less dense jets. The comparison suggests that the shock mechanism exciting the knots along the flows has the same efficiency in all the examined objects, and the observed differences are consistent with the different densities, and hence cooling rates, found in the various flows.
\end{abstract}

Key words. stars: circumstellar matter - infrared: ISM - ISM: Herbig-Haro objects - ISM: jets and outflows

\section{Introduction}

Spectral analysis is a powerful tool to investigate the nature and properties of astrophysical nebulae excited by shocks and/or energetic radiation. The diffuse matter in such objects produces a wealth of permitted and forbidden emission lines, whose excitation properties are reasonably well known. This in principle allows us to go back from the observed line intensities and line ratios to the physical conditions of the emitting material, which in turn provide essential information to test theoretical models and plan challenging observational programs, e.g. those involving interferometry. For quite a long time line diagnostics have been

* Based on observations collected at the European Southern Observatory, La Silla, Chile (ESO programmes 070.C-0396(A), 070.C.-0396(B)). used mainly to determine the gas electron density $\left(n_{\mathrm{e}}\right)$ and electron temperature $\left(T_{\mathrm{e}}\right)$ (see, e.g. Osterbrock 1994). The spectra of line emitting regions, however, contain much more information and the development of a number of new diagnostic techniques allow us to put, directly or indirectly, new important constraints on the gas physics. The study of stellar jets is one of the fields that has benefitted enormously from progress in spectral techniques. These collimated flows appear right from the birth of a star and indeed are believed to play a central role in the star formation process itself (see, e.g., Eislöffel et al. 2000b; Reipurth \& Bally 2001; Ray et al. 2003; Bacciotti et al. 2004). In fact, jets can help clear the circumstellar environment, thus setting a limit to the final central mass, and may even extract most of the excess angular momentum from the star/disk system (Bacciotti et al. 2002; Coffey et al. 2004; Woitas et al. 2005). Prototypical 
cases are the HH 34 and HH 111 jets in Orion (Reipurth \& Bally 2001), also studied in this work. All the observed features in stellar jets, such as bright knots along the flow and giant bow shocks, are believed to trace shocks that develop within these highly supersonic flows. Passing through the shock front, part of the bulk kinetic energy of the flow is turned into thermal motions, and the gas is suddenly heated to high temperatures $\left(10^{5} \mathrm{~K}\right.$ or more $)$, compressed, and partially ionised. This situation favours the collisional excitation of upper levels of atomic transitions. A number of radiative lines, whose type and strength are characteristic of shock excitation, are produced. These, in turn, radiatively cool down the gas to its original temperature. The whole process takes place in a limited region of space behind the shock front called the "cooling zone", whose length may vary from $10^{13}$ to about $10^{15} \mathrm{~cm}$, depending on the shock strength, the pre-shock conditions and the composition of the gas. The structure of radiatively cooling shocks has been widely studied in the past by several authors (see e.g. Hartigan et al. 1987; Hollenbach 1989; Hartigan et al. 1994; Hollenbach 1997; Flower et al. 2003; Hartigan 2003, and references therein). Within the cooling region individual physical quantities vary enormously, creating a stratification of excitation conditions that results in the production of different kinds of lines. In ground based observations at moderate spatial resolution, such as those presented here, the internal structure of the shock cooling zone remains spatially unresolved. Nevertheless, the stratification of such regions can be investigated spectrally by analysing the many different lines arising from different layers.

In the optical and Near Infrared (NIR) ranges used in this work one observes forbidden and permitted lines of abundant atomic and molecular species, such as $\mathrm{H}, \mathrm{He}, \mathrm{O}, \mathrm{S}, \mathrm{N}, \mathrm{Fe}, \mathrm{C}$, $\mathrm{Ca}, \mathrm{H}_{2}$ (Reipurth \& Bally 2001). To extract more information from these lines, several attempts have been made to use novel techniques by various groups. Böhm et al. (1980), Brugel et al. (1981) were among the first to examine the potential of combining the observed line ratios using low spatial resolution spectra integrated over the shock cooling zone. Hirth et al. (1997) examined long-slit spectra to study the spatial and kinematical properties of the forbidden emission line regions and micro-jets of a large sample of $\mathrm{T}$ Tauri stars. Intensities of atomic lines have been compared with the prediction of shock models in, e.g., Raga \& Böhm (1986), Hartigan et al. (1987), and Hartigan et al. (1994), indirectly inferring quantities such as the hydrogen ionisation fraction $x_{\mathrm{e}}$ and the intensity of the magnetic field in the pre-shock region. A new simple technique to measure $x_{\mathrm{e}}$ and $T_{\mathrm{e}}$ from the ratio of optical lines was first presented by Bacciotti et al. (1995), and subsequently refined in Bacciotti \& Eislöffel (1999, hereafter BE99). The method, referred to as the "BE" technique, is based on the fact that the gas emitting forbidden lines is collisionally excited, but no assumption is made regarding the heating agent. This is clearly an advantage if the results are used to validate a given thermal model. On the other hand, the method assumes that the emitting gas is at a single temperature, which is not true in the cooling region behind a shock front. In this regard, one should consider that the results of the BE technique are relevant to the region behind the shocks in which the employed lines have their peak emission (see the discussion and diagrams in BE99). As we show in this paper, the stratification of temperature and densities present in the entire cooling zone can be traced using a larger sample of lines appropriate for different excitation conditions.

A number of jets have been analysed with the BE technique, leading to the finding that jets are only partially ionised, with average $x_{\mathrm{e}}$ values between 0.01 and 0.6 . This procedure is much easier to apply than a grid of shock models (although it is of more limited application), and thus well suited for the analysis of big datasets, as those provided by high angular resolution observations (Bacciotti et al. 1999; Bacciotti 2002). The main advantage in determining $x_{\mathrm{e}}$, independently from the gas heating mechanism, is that by combining its value with the derived electron density one can estimate the total density, $n_{\mathrm{H}}$, a fundamental parameter of the jet that is critical in the various models.

Diagnostic techniques using both optical and NIR lines, on the other hand, provides important complementary information on jet parameters. In particular, the combination of optical and NIR [Fe II] lines gives an independent tool to determine $T_{\mathrm{e}}$ and $n_{\mathrm{e}}$, which does not rely on the choice of elemental abundances, in the more compressed and cooler post-shock layer where these lines are excited (Nisini et al. 2002; Pesenti et al. 2003; Hartigan et al. 2004). In addition, the $\mathrm{NIR}_{2}$ lines provide a means to probe the molecular component of shock excited gas, which may give a significant contribution to the gas cooling in low velocity, magnetized shocks (Eislöffel et al. 2000a; Giannini et al. 2004).

Very recently, we have investigated the advantages of a combined optical and NIR spectral analysis of the HH 1 jet (Nisini et al. 2005) using a variety of diagnostic lines over a wide wavelength range (from 0.6 to $2.2 \mu \mathrm{m}$ ). The adopted procedure turned out to be extremely powerful for constructing detailed physical maps of the stratified media in the beams of stellar jets. In this paper, we analyse a large sample of "classical" stellar jets, located in the Orion and Vela star formation regions, with the same technique. We obtain their basic parameters, as well as important quantities derived from them, such as the mass flux rates. We analyse the variations of the parameters both behind each shock, i.e. depending on the tracers, and along the jet, i.e. as a function of the distance from the emitting source. We then discuss the differences/similarities among jets having different properties and ages. This information can in turn be used for the selection of suitable candidates for high angular resolution observations.

This paper is organized as follows: we describe our observations in Sect. 2 and briefly recall our diagnostic procedure in Sect. 3, adding a detailed discussion on the choice of the best set of elemental abundances, an issue that was not examined in our previous papers. In Sect. 4 we describe the results obtained for each target in the sample, and we discuss them in Sect. 5 together with the derivation of the depletion of refractory elements and the mass and linear momentum flux rates. Section 6 summarizes our findings.

\section{Observations}

We observed a sample of classical protostellar jets $(\mathrm{HH} 34$, HH 111, HH 83, HH 73, HH 24 C/E, HH 24 J) in the spectral range from $6015 \AA$ to $2.52 \mu \mathrm{m}$. We acquired the optical spectra (6015-10320 A) at the ESO 3.6-m telescope equipped with the spectrograph EFOSC2. Infrared spectra were taken at the NTT with SofI using both the blue grism (IR-GB: 0.95-1.64 $\mu \mathrm{m}$ ) and the red grism (IR-GR: $1.53-2.52 \mu \mathrm{m}$ ). In both cases the spectra were taken aligning the slit parallel to the jet axis. The position angles are: $166^{\circ}$ for $\mathrm{HH} 34,277^{\circ}$ for $\mathrm{HH} 111,299^{\circ}$ for $\mathrm{HH} 83$, $303^{\circ}$ for $\mathrm{HH} 73,334^{\circ}$ for $\mathrm{HH} 24 \mathrm{C} / \mathrm{E}$, and $309^{\circ}$ for $\mathrm{HH} 24 \mathrm{~J}$. The observational settings were chosen to obtain, as far as possible, homogeneous spectra in the two wavelength ranges, in order to be able to apply a combined optical/NIR analysis. The observations were all carried out over a short period of time (7-8 and 11-12 January 2003, for the optical and IR observations, respectively). In this reagard, note that jet parameters change over 
timescales not shorter than a few years and probably decades in most cases. A $1^{\prime \prime}$ width slit was used for both the optical and the IR spectra, in order to cover the same section of the jet and to have similar spectral resolution $(R \sim 600)$. Moreover, the spatial scale of the two cameras is comparable (0.'314/pixel for EFOSC2 and 0.'288/pixel for SofI). The integration times for the EFOSC2 observations were $1800 \mathrm{~s}$ for HH 111, HH 34, HH 73 and $5400 \mathrm{~s}$ for $\mathrm{HH} 83, \mathrm{HH} 24 \mathrm{C} / \mathrm{E}, \mathrm{HH} 24 \mathrm{~J}$. In the infrared the exposure times for each of the two SofI grisms are: $1800 \mathrm{~s}$ for HH 111 and $\mathrm{HH}$ 34, $1200 \mathrm{~s}$ for $\mathrm{HH} 83$ and $\mathrm{HH} 24 \mathrm{C} / \mathrm{E}, 600 \mathrm{~s}$ for HH $24 \mathrm{~J}, 800 \mathrm{~s}$ in the IR-GB and $400 \mathrm{~s}$ in the IR-GR for HH 73. In addition, telluric and spectro-photometric standards were observed in order to correct for the atmospheric spectral response and to flux-calibrate the spectra respectively. The wavelength calibrations were performed using a helium-argon lamp in the optical and a xenon lamp in the infrared. The data reduction was done using standard IRAF tasks. We obtained for each jet three individually calibrated spectra in the optical, IR-GB and IR-GR ranges. For each knot, a single spectrum from $6015 \AA$ to $2.52 \mu \mathrm{m}$ was then formed as follows: (i) the same spatial reference was defined in each of the three spectra; (ii) a knot length was set by comparing the spatial profiles along different lines with our images acquired through [SII] and $\mathrm{H}_{2}$ narrow-band filters and with high angular resolution images from the literature. The same knot size was then adopted in all three spectral domains; (iii) we then extracted from each spectral image, and for each knot, the corresponding spectrum integrating over the size defined above; (iv) for each knot, the three obtained spectra were inter-calibrated measuring the fluxes of the lines located in the overlapping spectral regions (the [CI]9850 $\AA$ line flux in the optical/IR-GB and the [FeII]1.64 $\mu \mathrm{m}$ line in the IR-GB/IR-GR).

\section{Description of the combined optical/NIR spectral analysis}

In Sect. 3.1 the principles underlying our diagnostic techniques are briefly recalled. In Sect. 3.2 we present a critical discussion on the adoption of a given set of elemental abundances, which is necessary for our analysis.

\subsection{Derivation of the physical conditions along the jet beam}

Thanks to the many lines observed over the wide spectral range covered by our EFOSC2/SofI spectra, and using together optical (BE99) and infrared (Nisini et al. 2002; Pesenti et al. 2003) diagnostic techniques, we have been able to determine the parameters describing the stratified medium behind each shock even if the knots along the jets are not spatially resolved.

Firstly, the visual extinction, $A_{\mathrm{V}}$, has to be determined to deredden the spectra and be able to combine lines that are far apart in wavelength. $A_{\mathrm{V}}$ can be found using pairs of distant lines coming from the same upper level of a single ion and assuming a reddening law. Suitable choices in our case are the [Fe II] $1.64 \mu \mathrm{m} / 1.25 \mu \mathrm{m}$ or the [Fe II] $1.64 \mu \mathrm{m} / 1.32 \mu \mathrm{m}$ ratios. We tried to calculate $A_{\mathrm{V}}$ from both these ratios and using different sets of Einstein coefficients (Nussbaumer \& Storey 1988; Quinet et al. 1996; Smith \& Hartigan 2006). With any of the above sets the [Fe II] $1.64 \mu \mathrm{m} / 1.25 \mu \mathrm{m}$ ratios give higher values of $A_{\mathrm{V}}$ than those inferred through using [Fe II] $1.64 \mu \mathrm{m} / 1.32 \mu \mathrm{m}$. Nisini et al. (2005) pointed out that the [Fe II] $1.64 \mu \mathrm{m} / 1.25 \mu \mathrm{m}$ ratio, compared with the theoretical value adopting the radiative rates from Nussbaumer \& Storey (1988), provides extinction values which are too high, inconsistent with determinations in the optical and with model predictions (see their Appendix B). We find that the [Fe II] $1.64 \mu \mathrm{m} / 1.25 \mu \mathrm{m}$ ratio empirically estimated by Smith \& Hartigan (2006) from the spectrum of P Cygni produces even higher $A_{\mathrm{V}}$ values. On the other hand, Smith \& Hartigan (2006) derive a [Fe II]1.64 $\mu \mathrm{m} / 1.32 \mu \mathrm{m}$ theoretical ratio very similar to that of Nussbaumer \& Storey (1988), which gives an $A_{\mathrm{V}}$ more consistent with other independent determinations (Nisini et al. 2005). Thus we adopted the $A_{\mathrm{V}}$ value derived from the [Fe II] $1.64 \mu \mathrm{m} / 1.32 \mu \mathrm{m}$ ratio and the Nussbaumer \& Storey (1988) coefficients as in Nisini et al. (2005). All the spectra were then corrected for extinction using a standard dereddening procedure (Draine 1989) and an interpolation of the extinction law derived by Rieke \& Lebofsky (1985) for the near-IR bands.

The electron density, $n_{\mathrm{e}}$, in the region of optical emission, was derived from the $[\mathrm{S}$ II $] \lambda 6716 \AA /[\mathrm{S} \mathrm{II}] \lambda 6731 \AA$ ratio (Osterbrock 1994). Since in some of the knots the [S II] $\lambda \lambda 6716$, 6731 doublet was not resolved sufficiently to separately measure the line fluxes, we used data taken two years before by our group using EFOSC2 at higher spectral resolution (Medves et al., in prep.). Intervening proper motions of the jet knots can be neglected for the purposes of our analysis. Then, using the BE technique (see BE99), that employs selected optical transitions of $\mathrm{S}^{+}, \mathrm{O}^{0}$, and $\mathrm{N}^{+}$, we derived the electron temperature, $T_{\mathrm{e}}$, and the hydrogen ionisation fraction, $x_{\mathrm{e}}$. Since in determining these quantities one uses ratios between different species (namely [N II] $\lambda \lambda(6548+6583) /[\mathrm{OI}] \lambda \lambda(6300+6363)$ and $[\mathrm{S} \mathrm{II}] \lambda \lambda(6716+6731) /[\mathrm{OI}] \lambda \lambda(6300+6363))$, the technique requires the adoption of a given set of elemental abundances. This issue is examined in detail in the next section. Finally, from the values inferred for $n_{\mathrm{e}}$ and $x_{\mathrm{e}}$, we derived a gross estimate of the total hydrogen density $\left(n_{\mathrm{H}}=n_{\mathrm{e}} / x_{\mathrm{e}}\right)$. Refinements to this density estimate are discussed in Sect. 5. Also, as mentioned in the Introduction, we stress that the results of the BE technique are only relevant to the region of the cooling zone behind the shock front where the considered optical lines emit the most (see BE99).

The errors that affect the parameters obtained through the $\mathrm{BE}$ diagnostic are due to measurement errors of the line fluxes (which depend on the signal-to-noise ratio) and the uncertainty in the determination of $A_{\mathrm{V}}$ which is used to deredden the line fluxes. To determine $x_{\mathrm{e}}$ and $T_{\mathrm{e}}$ the uncertainty in $n_{\mathrm{e}}$ values also has to be taken into account. On the other hand, since the lines used in this diagnostic are very close in wavelength the main source of uncertainty is the measurement error. In general, for the brightest knots, where the signal-to-noise is high $(S / N>10)$, the errors are $<5 \%$ for $n_{\mathrm{e}},<15 \%$ for $x_{\mathrm{e}}$ values and $<10 \%$ for $T_{\mathrm{e}}$.

[Fe II] lines give a completely independent way to determine the physical conditions of the gas. From the ratios $[\mathrm{Fe}$ II] $1.64 \mu \mathrm{m} / 1.53 \mu \mathrm{m}$ and [Fe II] $1.64 \mu \mathrm{m} / 1.60 \mu \mathrm{m}$ one can infer the electron density $n_{\mathrm{e}}$ in the region of Fe emission. The ratios between optical and infrared [Fe II] lines can be used to find the temperature in the same region. The relevant ratios for this goal, are those of the $1.64 \mu \mathrm{m}$ line with different transitions between $8000 \AA$ and $1 \mu \mathrm{m}$ originating from the $a^{4} \mathrm{P}$ term (Nisini et al. 2002; Pesenti et al. 2003). As we will show, [Fe II] lines trace the cooler and denser gas located further from the shock front than the zone of optical line emission, and thus probe a region where the emitting material is more compressed (see Hollenbach 1997).

In order to check if there is an even denser component in the knots we used the CaII $\lambda 8540 /[\mathrm{Ca}$ II $] \lambda 7290$ and $[\mathrm{Fe}$ II $] \lambda 7155 / \lambda 8617$ ratios. The theoretical values of 
Ca II ratios for three different values of the temperature $(T=$ $5000 \mathrm{~K}, T=10000 \mathrm{~K}, T=15000 \mathrm{~K}$ ) were computed with a five-level statistical equilibrium code (radiative transition rates from NIST; collisional rates from Mendoza 1983; and Chidichimo 1981). This code assumes purely collisional excitation. The observed ratio [Ca II] $\lambda 7290 / \lambda 7324, \sim 1.5$ in all the cases, is consistent with the ratio expected for collisional excitation. Such a ratio is however also consistent with fluorescence pumping of the $4 p$ levels giving rise to the $\mathrm{Ca}$ II $\mathrm{H}$ and K UV lines, followed by cascade to the $3 \mathrm{~d}$ level (Hartigan et al. 2004). Hartigan et al. (2004) estimated a neglegible fluorescence pumping rate with respect to the collisional rate in the $\mathrm{Ca}$ II excitation of the HN Tau jet observed at $10 \mathrm{AU}$ from the source. We assume that this is the case also for the jets in our sample, since we are dealing with jets located at large distance from the driving source, where no significant UV field is expected. The observed values of [Fe II] ratios, instead, were compared with prediction diagrams of Bautista \& Pradhan (1996), in order to determine the electron density. These diagrams plot the predicted $[\mathrm{Fe}$ II] ratios versus the electron density for a fixed temperature (we considered the curve for $T=10^{4} \mathrm{~K}$ ).

Finally, to derive the gas physical conditions in the region of molecular emission, we use $\mathrm{H}_{2}$ lines diagnostics. In particular, from the rotational diagrams we estimate the temperature and the total column density of the molecular gas (see Nisini et al. 2002, for more details).

\subsection{Choice of elemental abundances}

As discussed in Sect. 3.1, to estimate the ionisation fraction, $x_{\mathrm{e}}$, and the temperature, $T_{\mathrm{e}}$, one has to assume the relative abundances of $\mathrm{S}, \mathrm{O}, \mathrm{N}$ used in the diagnostic. As a consequence, the derived values may depend on the adopted set of abundances, an issue that has to be investigated in more detail. For example, in Nisini et al. (2005) we performed our diagnostic analysis adopting solar abundances from Grevesse \& Sauval (1998). The majority of our targets, however, are located in the Orion Cloud. Moreover, there are many lines of evidence that suggest Solar System abundances may not even be representative of the local ISM 4.6 Gyr ago, at the time the Sun formed (Wilson \& Rood 1994). It follows that solar abundances may not be adequate to study the properties of the jets in our sample. To elucidate the situation, we test the effect of abundance variation on our diagnostic results. The abundance sets we consider are: (i) the solar abundances determined by Grevesse \& Sauval (1998, G\&S 98); (ii) the most recent determinations of the solar abundances by Asplund et al. (2005, A 05); (iii) the abundances adopted in BE99 (taken from Hartigan et al. 1987) with the aim of testing agreement between the BE diagnostics and shock models; and (iv) the abundances determined for the interstellar gas in the Orion Nebula by Esteban et al. (2004, E 04). The values of elemental abundances of $\mathrm{S}, \mathrm{O}, \mathrm{N}$ in these sets are summarized in Table 1.

We computed the values of $x_{\mathrm{e}}$ and $T_{\mathrm{e}}$ for the HH 111 jet assuming the above sets of abundances. The results are shown in the upper panels of Fig. 1. The figure shows that with the Orion abundances of Esteban et al. (2004) we obtain the highest values of $x_{\mathrm{e}}$ and $T_{\mathrm{e}}$ from our analysis. On the other hand, these values are within $15 \%$ of those obtained using the recent solar abundances determination of Asplund et al. (2005), since the abundances of the elements involved in the diagnostics are quite similar in the two sets. Moreover, the values of $x_{\mathrm{e}}$ and $T_{\mathrm{e}}$ found assuming the solar abundances by Grevesse \& Sauval (1998) are again in good agreement with the previous ones.
Table 1. Abundance sets.

\begin{tabular}{cccc}
\hline \hline Abundance set & $\begin{array}{c}\mathrm{S} / \mathrm{H} \\
\left(10^{-5}\right)\end{array}$ & $\begin{array}{c}\mathrm{O} / \mathrm{H} \\
\left(10^{-4}\right)\end{array}$ & $\begin{array}{c}\mathrm{N} / \mathrm{H} \\
\left(10^{-5}\right)\end{array}$ \\
\hline (i) Solar (G\&S 98) $^{a}$ & 2.14 & 6.76 & 8.32 \\
(ii) Solar (A 05) $^{b}$ & 1.38 & 4.57 & 6.03 \\
(iii) Adopted in BE99 $^{c}$ & 1.6 & 6.0 & 11.0 \\
(iv) Orion (E 04) $^{d}$ & 1.66 & 4.47 & 5.37 \\
\hline
\end{tabular}

${ }^{a}$ Grevesse \& Sauval (1998); ${ }^{b}$ Asplund et al. (2005); ${ }^{c}$ Bacciotti \& Eislöffel (1999); ${ }^{d}$ Esteban et al. (2004).
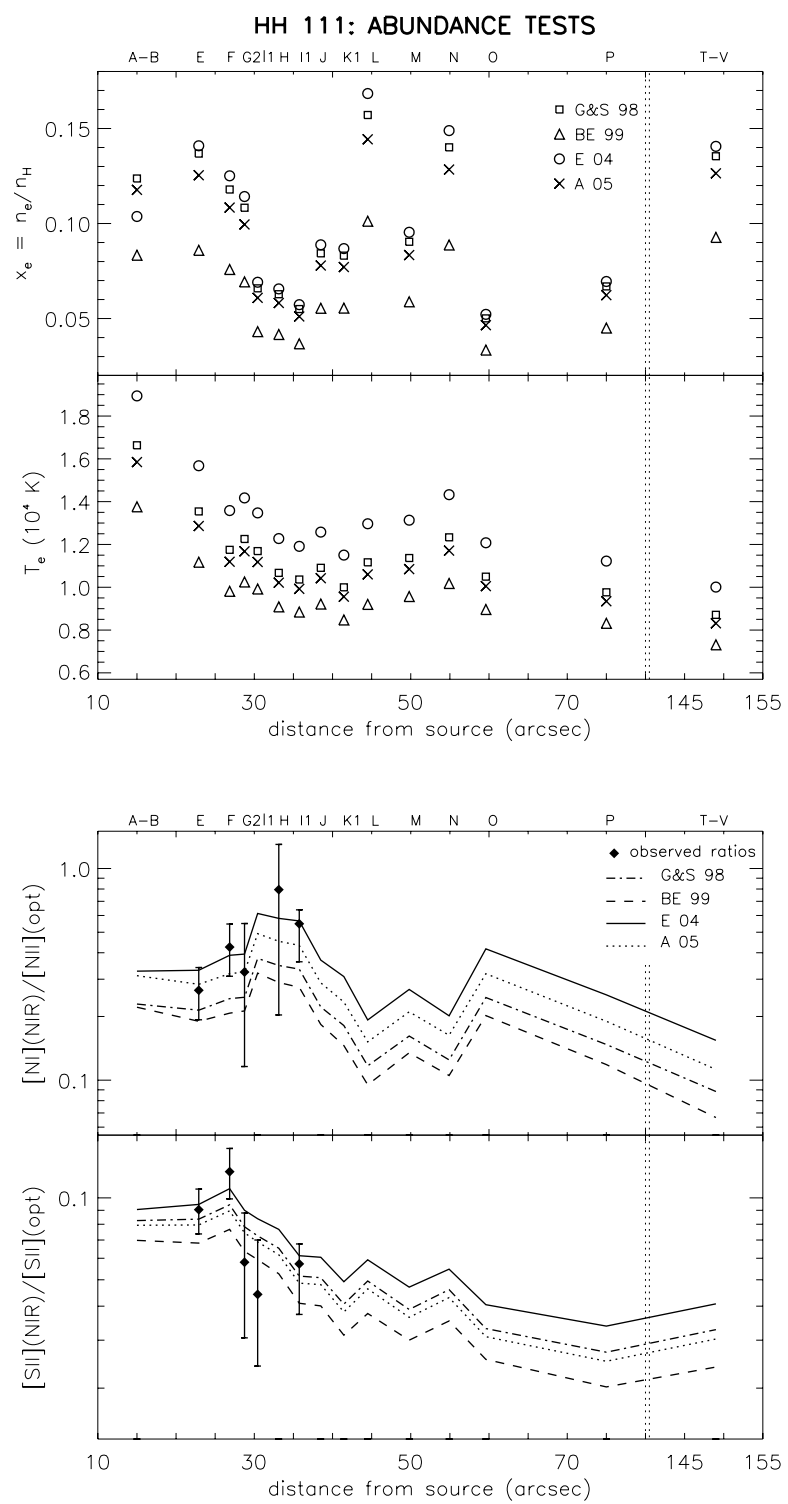

Fig. 1. Variations in results using the BE technique and different adopted elemental abundance sets. The HH 111 jet is used for test purposes. Upper panel: values of the ionisation fraction $x_{\mathrm{e}}$ and electron temperature $T_{\mathrm{e}}$ in each jet knot assuming: solar abundances from Grevesse \& Sauval (1998) (squares) and Asplund et al. (2005) (crosses); abundances adopted in BE99 (triangles); Orion abundances from Esteban et al. (2004) (circles). Lower panel: comparison between observed values of the $[\mathrm{NI}](1.04 \mu \mathrm{m}) /[\mathrm{N} \mathrm{II}](6583 \AA)$ and $[\mathrm{S}$ II $](1.02+1.03 \mu \mathrm{m}) /[\mathrm{S}$ II $](6716+6731 \AA)$ ratios and theoretical predictions for the various abundance sets.

The reason here is somewhat different: despite the Orion E 04 set and the solar G\&S 98 set having different abundances, the 
Table 2. Physical parameters of the jets averaged over the brightest knots.

\begin{tabular}{cc|cccc|cc}
\hline \hline & & \multicolumn{4}{|c|}{ From $\mathrm{O}^{0} / \mathrm{S}^{+} / \mathrm{N}^{+}$lines } & \multicolumn{2}{c}{ From Fe $^{+}$lines } \\
Jet & $A_{\mathrm{V}}$ & $n_{\mathrm{e}}$ & $x_{\mathrm{e}}$ & $T_{\mathrm{e}}$ & $n_{\mathrm{H}}$ & $n_{\mathrm{e}}$ & $T_{\mathrm{e}}$ \\
& $(\mathrm{mag})$ & $\left(10^{3} \mathrm{~cm}^{-3}\right)$ & & $\left(10^{3} \mathrm{~K}\right)$ & $\left(10^{3} \mathrm{~cm}^{-3}\right)$ & $\left(10^{3} \mathrm{~cm}^{-3}\right)$ & $\left(10^{3} \mathrm{~K}\right)$ \\
\hline HH 111 & $2.0-0$ & 1.0 & 0.10 & 13.0 & 11.3 & 2.6 & 7.3 \\
HH 34 & $7.1-1.3$ & 0.8 & 0.04 & 13.8 & 16.2 & 1.8 & 5.8 \\
HH 83 & - & 0.5 & 0.38 & 17.5 & 0.9 & - & - \\
HH 73 & - & 0.6 & 0.31 & 17.5 & 1.7 & - & - \\
HH 24 C/E & - & 0.4 & 0.32 & 19.3 & 1.3 & - & - \\
\hline
\end{tabular}

ratios $\mathrm{O} / \mathrm{N}$ and $\mathrm{S} / \mathrm{O}$ used in the analysis turn out to be similar. On the other hand, the values inferred from the abundance set adopted in BE99 show differences of up to $40 \%$ for $x_{\mathrm{e}}$ and up to $25 \%$ for $T_{\mathrm{e}}$. We also tested the results obtained with the different sets using the observed ratios $[\mathrm{N} \mathrm{I}](1.04 \mu \mathrm{m}) /[\mathrm{N}$ II $](6583 \AA)$ and $[\mathrm{S} \mathrm{II}](1.02+1.03 \mu \mathrm{m}) /[\mathrm{S} \mathrm{II}](6716+6731 \AA)$, that do not depend on abundances, but do depend on the derived $x_{\mathrm{e}}$ and $T_{\mathrm{e}}$. Comparing observed and theoretical values (see the lower panels of Fig. 1) we find that the abundance sets (i), (ii) and (iv) are all consistent with the observations, while the abundances adopted in BE99, that yield the lowest theoretical values for these ratios, deviate more.

Following the above results, we chose to adopt the abundance set by Esteban et al. (2004) for the jets in our sample located in the Orion cloud. For HH 73, located in Vela, we tentatively assume the same, since no abundance determination exists for Vela.

\section{Diagnostic results: basic physical parameters of the examined jets}

In this section we describe our determination of basic physical parameters, i.e., density, temperature and ionisation fraction, in each jet knot, obtained from the application of the combined optical/NIR technique to our sample. The best data were obtained for the HH 111 jet, allowing us to get very accurate values in this case. Parameters for HH 34 also appear to be very reliable. HH 111 and $\mathrm{HH} 34$ are very well known $\mathrm{HH}$ objects and they have been investigated previously both in the optical wavelength range (Eislöffel \& Mundt 1992; Morse et al. 1993; Noriega-Crespo et al. 1993; Hartigan et al. 1994; Eislöffel \& Mundt 1997; Reipurth et al. 1997; BE99; Hartigan et al. 2001; Reipurth et al. 2002; Raga et al. 2002) and in the near-infrared (Stapelfeldt et al. 1991; Stanke et al. 1998; Davis et al. 2001; Nisini et al. 2002). Our purpose here is to combine the information coming from the different tracers emitting in the two wavelength ranges, in order to obtain a detailed physical map of the stratified medium in each unresolved cooling zone along these outflows. The fainter objects $\mathrm{HH} 83, \mathrm{HH} 73$, and $\mathrm{HH} 24$, instead, were either not seen in the IR (HH 73) or were visible only in one or two knots (HH 83 and HH 24). Thus for these jets we only apply the optical diagnostic to obtaine a description of the physical conditions of the gas emitting in this range. Note that, although it is not possible to estimate the extinction when the [Fe II] lines are not detected, we can still use the optical diagnostic since the lines involved in the BE technique are very near in wavelength. A summary of our results is given in Table 2, but note that the derived physical parameters are averages for the brightest knots.

\section{1. $H H 111$}

One of the best known stellar jets is HH 111. This outflow, located in the L1617 cloud in Orion $(D=460 \mathrm{pc})$, is powered by the young star IRAS $05491+0247$ and extends over several parsecs (Reipurth et al. 1997). The exciting source is deeply embedded in the parental molecular cloud core (in our spectra the source is detected only in the infrared $K$-band) and the base of the jet is not visible at optical wavelengths. The main visible jet emerges from the cloud only $15^{\prime \prime}$ from the source and is blueshifted. Adopting the nomenclature of Reipurth et al. (1997), this lobe consists of a long chain of knots, with a bow-like morphology, observable in the optical from 15" (knot A-B) to 80" $($ knot $\mathrm{P})$ and terminates with a bow shock $($ knot $\mathrm{T}-\mathrm{V})$ at a distance of $150^{\prime \prime}$ from the driving source. The red-shifted lobe is almost completely obscured, with only knot ZL visible in the optical, while knots ZO and ZV (symmetric to knots $\mathrm{O}$ and $\mathrm{T}-\mathrm{V}$ in the blue lobe) have been observed only in the near-infrared.

Several authors have investigated the physical properties of this flow in the past. Morse et al. (1993) measured the electron density along the jet from the [S II] line ratio and presented a complete list of the lines emitted in the optical range (see also Noriega-Crespo et al. 1993). Hartigan et al. (1994) estimated a value of the ionisation fraction averaged along the entire flow comparing the observed line ratios with the prediction of shock models, and derived an estimate of the average mass loss rate. Internal gas kinematics has been investigated by Hartigan et al. (2001) and Raga et al. (2002). In particular, in the first paper proper motions of the knots were determined, from which shock velocities of about $40 \mathrm{~km} \mathrm{~s}^{-1}$ were derived, in agreement with the type of line observed. In the infrared wavelength range, Davis et al. (2001) studied the kinematics of the gas emitting in $\mathrm{H}_{2}$, while an analysis of $\mathrm{H}_{2}$ and [Fe II] lines along the jet has been presented in Nisini et al. (2002).

In this paper we apply our combined optical/NIR analysis to the knots in the blue lobe from $15^{\prime \prime}$ outwards, where both optical and infrared lines are observed (except for knot $\mathrm{T}-\mathrm{V}$ that is out of the frame in the IR spectra). In Fig. 2 the spatial normalized distribution of the optical [S II] line is compared with the corresponding [Fe II] and $\mathrm{H}_{2} 2.12 \mu \mathrm{m}$ distributions. [S II] and [Fe II] lines have similar shape and brightness, while the $\mathrm{H}_{2}$ line is fainter and has a different spatial distribution. In particular the emission of [S II] and [Fe II] lines is maximum at knot $\mathrm{E}$ with a flux of $\sim 4 \times 10^{-14} \mathrm{erg} \mathrm{s}^{-1} \mathrm{~cm}^{-2}$, while the maximum $\mathrm{H}_{2}$ emission is reached in knot $\mathrm{P}\left(\sim 1.4 \times 10^{-14} \mathrm{erg} \mathrm{s}^{-1} \mathrm{~cm}^{-2}\right)$. Moreover, the $[\mathrm{S} \mathrm{II}]$ and [Fe II] line intensities decrease with distance from the source, while $\mathrm{H}_{2}$ emission is stronger in the outer bows ( $\mathrm{L}$ and P). Such behavior, also noted by Nisini et al. (2002), can be explained by the fact that [Fe II] and [S II] emission is expected to be excited from dissociative J-shocks $\left(v_{\mathrm{s}}>30-40 \mathrm{~km} \mathrm{~s}^{-1}\right)$ at the apex of the bows, while the $\mathrm{H}_{2}$ emission arises from the bow wings where the transverse component of the velocity gives 


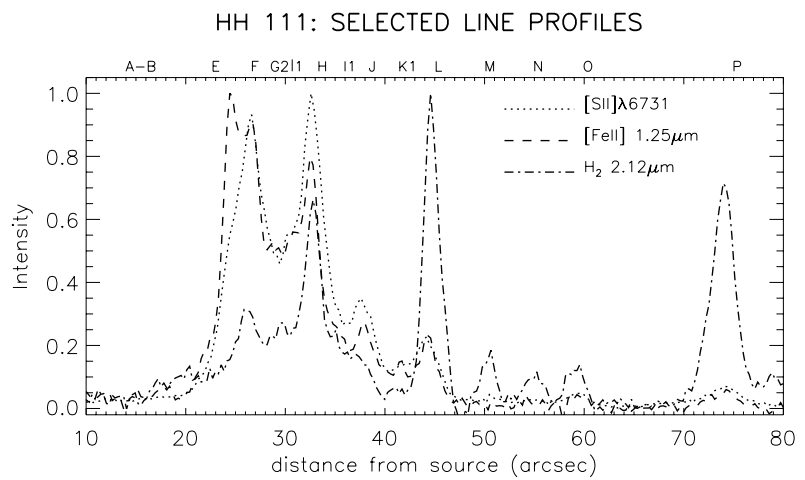

Fig. 2. Spatial profiles of the $[\mathrm{S} \mathrm{II}],[\mathrm{Fe} I \mathrm{II}]$, and $\mathrm{H}_{2} 2.12 \mu \mathrm{m}$ lines along the HH 111 jet. The zero point of the spatial scale is the source HH 111 IRS (RA(2000): 0551 46.1, Dec(2000): +02 48 30).

rise to slower C-shocks $\left(v_{\mathrm{s}}<30 \mathrm{~km} \mathrm{~s}^{-1}\right)$ that prevent $\mathrm{H}_{2}$ dissociation (Davis et al. 1996; Hollenbach 1997; Davis et al. 1999). Observational confirmations for such an $\mathrm{H}_{2}$ distribution is seen for example in the HH 211 flow (Gueth et al. 1999). In this scenario the fact that the $([\mathrm{Fe} \mathrm{II}],[\mathrm{S} \mathrm{II}]) / \mathrm{H}_{2}$ observed ratio decreases with distance from the source would be consistent with the suggestion that in the outer bows there is a prevalence of nondissociative slow C-shocks, since the ambient density becomes higher or comparable to the jet density (Hollenbach 1989).

In order to apply the diagnostics to line ratios, we first determined the visual extinction towards all the knots. As expected, the extinction is quite low outside the dense core around the source, being $A_{\mathrm{V}} \sim 2$ mag for the innermost visible knot $(\mathrm{A}-\mathrm{B})$, and decreasing to $A_{\mathrm{V}} \sim 0$ mag for the farthest knots (from I1 to $\mathrm{T}-\mathrm{V})$. These values are much smaller than those derived in Gredel et al. (1993) and in Nisini et al. (2002), who adopted the $1.64 / 1.25 \mu \mathrm{m}$ ratio for the $A_{\mathrm{V}}$ determination, and smaller but more similar, at least for the inner knots, to the values derived from the Balmer decrement (Morse et al. 1993; Noriega-Crespo et al. 1993). As explained in Sect. 3.1 and, more in detail, in the appendix of Nisini et al. (2005), here we assume that the $1.64 / 1.32 \mu \mathrm{m}$ ratio yields a more reliable estimate of the reddening than the $1.64 / 1.25 \mu \mathrm{m}$ ratio when the Nussbaumer \& Storey (1988) Einstein coefficients are considered.

After correcting line fluxes for reddening, we derive the values of the physical parameters, $n_{\mathrm{e}}, x_{\mathrm{e}}, T_{\mathrm{e}}$, and $n_{\mathrm{H}}$, separately for the different lines considered, for each knot along the jet, with the procedures described in Sect. 3.1. The results are shown in Fig. 3. The electron density $n_{\mathrm{e}}$ from [S II] lines (filled circles) increases from $\sim 7.5 \times 10^{2} \mathrm{~cm}^{-3}$ in the first knot, $\mathrm{A}-\mathrm{B}$, to $\sim 10^{3} \mathrm{~cm}^{-3}$ in the brightest optical knots, reaching a maximum value of $\sim 2 \times 10^{3} \mathrm{~cm}^{-3}$ in $\mathrm{F}$. Then it slowly decreases with distance from the source down to $n_{\mathrm{e}} \sim 0.7 \times 10^{2} \mathrm{~cm}^{-3}$ at knot $\mathrm{P}$, and finally presents another local maximum in knot $\mathrm{T}-\mathrm{V}$ $\left(n_{\mathrm{e}} \sim 5 \times 10^{2} \mathrm{~cm}^{-3}\right)$. These values agree with the average electron density of $900 \mathrm{~cm}^{-3}$ found by Hartigan et al. (1994), while they are of a factor of two lower with respect to the values found by Morse et al. (1993). The electron temperature, $T_{\mathrm{e}}$, derived from the optical lines is similar in all the various knots, i.e. cooling regions along the jet, being on average $\sim 1.3 \times 10^{4} \mathrm{~K}$, if exception is made for the first noisy point at the base of the jet $\left(T_{\mathrm{e}} \sim 1.9 \times 10^{4} \mathrm{~K}\right)$.

The values of the hydrogen ionisation fraction $x_{\mathrm{e}}$, in each knot, and averaged over the region of the cooling zones where the considered optical lines emit the most, vary from a minimum of 0.05 to 0.17 . In our determination, $x_{\mathrm{e}}$ decreases steadily
HH 111 DIAGNOSTICS

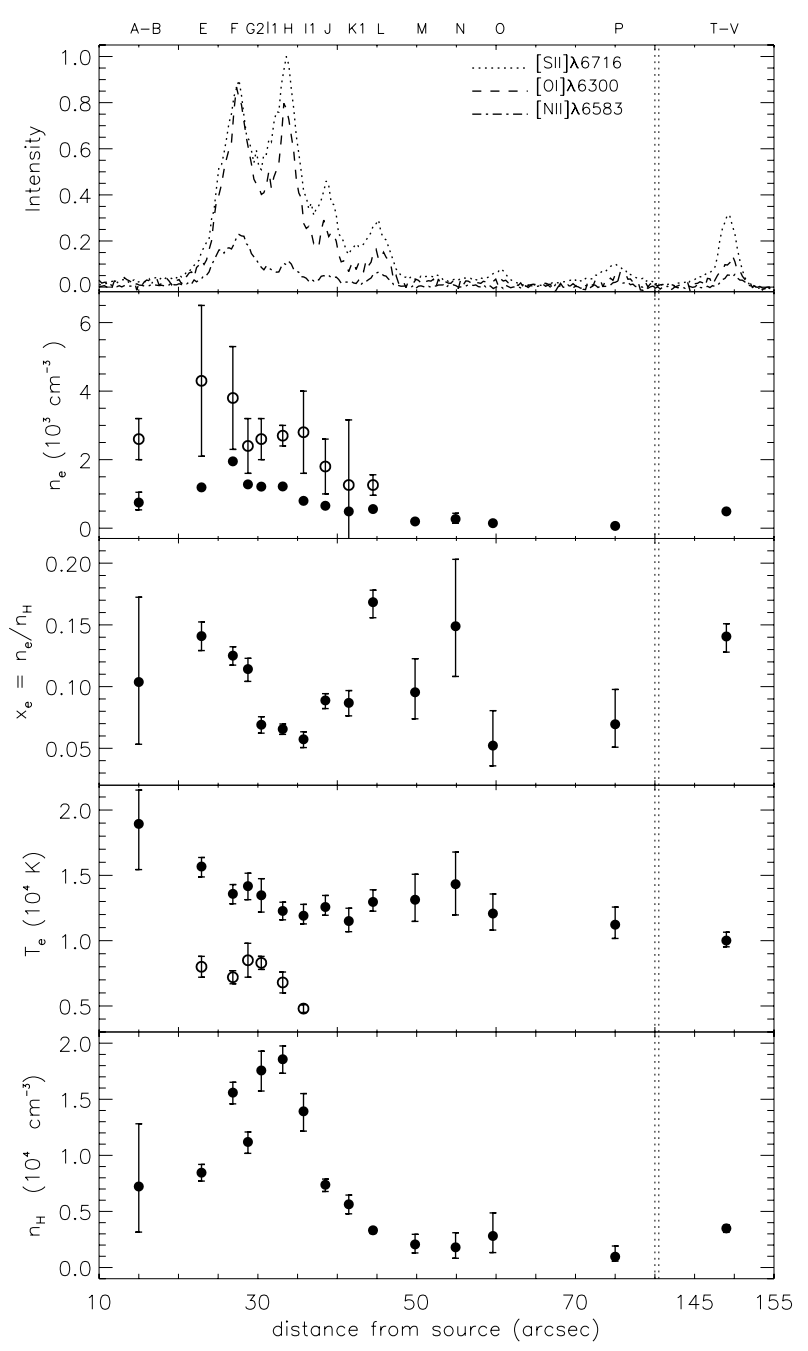

Fig. 3. Variation of the derived physical parameters along the HH 111 jet. From top to bottom panel: intensity profiles of the optical lines, the electron density, $n_{\mathrm{e}}$, in units of $10^{3} \mathrm{~cm}^{-3}$, the ionisation fraction, $x_{\mathrm{e}}$, the temperature, $T_{\mathrm{e}}$, in units of $10^{4} \mathrm{~K}$ and the total density, $n_{\mathrm{H}}$, in units of $10^{4} \mathrm{~cm}^{-3}$. The open circles are the values derived from the $[\mathrm{Fe}$ II] lines, while the filled circles are parameters inferred from the optical $\mathrm{S}^{+}, \mathrm{N}^{+}$and $\mathrm{O}^{0}$ lines using the $\mathrm{BE}$ technique. Note that the zero point of the spatial scale is the driving source HH 111 IRS (see Fig. 2).

from knot $\mathrm{E}$ to knot $\mathrm{K} 1$, like the electron density, but it increases again at knot $\mathrm{L}$ to a value of 0.17 . In the subsequent faint region of the beam the ionisation fraction appears to decrease again, although the accuracy of the derivation is much lower here. Finally another local maximum is retrieved at the isolated knot $\mathrm{T}-\mathrm{V}$ very far from the source, where $x_{\mathrm{e}}$ is 0.14 . The lower limit found for the jets brightest knots is similar to the one $\left(x_{\mathrm{e}}=0.052\right)$ derived by Hartigan et al. (1994) by comparing the observed $[\mathrm{N} \mathrm{II}] /[\mathrm{OI}]$ ratio, which is sensitive to $x_{\mathrm{e}}$, to the predictions of low velocity shock models. In other knots, however, we find higher values that are difficult to justify, as a shock with effective velocity of $30-40 \mathrm{~km} \mathrm{~s}^{-1}$ hardly produces an ionisation fraction greater than a few percent (see Fig. 1 in Hartigan et al. 1994). The contradiction can be reconciled if one considers that the shock fronts in the jet beam advance in the wake of the previous shock, and thus move in a medium that has been already pre-ionised. We note that a substantial level of hydrogen 


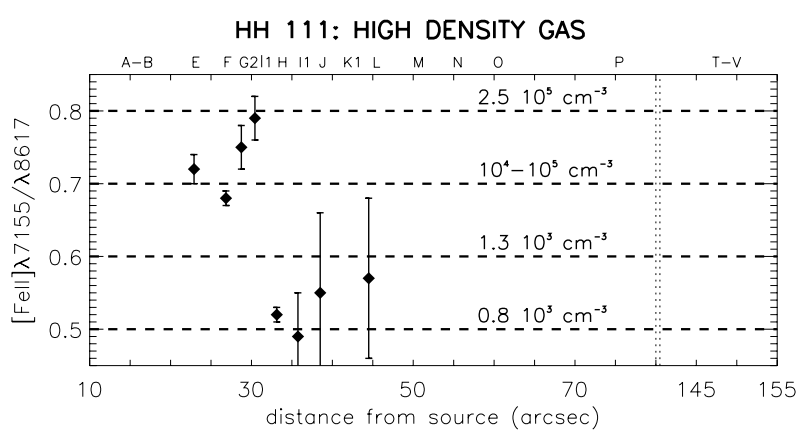

Fig. 4. Comparison between the observed $[\mathrm{Fe}$ II $] \lambda 7155 /[\mathrm{Fe}$ II] $] \lambda 8617 \mathrm{ra}-$ tios (diamonds) and the theoretical curves (dashed lines) computed by Bautista \& Pradhan (1996) for different values of the electron density, $n_{\mathrm{e}}$, and a fixed temperature of $10^{4} \mathrm{~K}$. This ratio traces a dense component in the jet beam $\left(n_{\mathrm{e}}\right.$ up to $2.5 \times 10^{5} \mathrm{~cm}^{-3}$ in the knots close to the source). Note that the zero point of the spatial scale is the driving source HH 111 IRS (see Fig. 2).

ionisation can persist between two consecutive shocks because the recombination time is slow in the rarefied jet gas. It can be shown that with the typical electron densities and bulk velocities of the jet material, the recombination time is of the same order of the crossing time of the entire bright section of the jet (see BE99). On the other hand, this explanation may not apply to the relatively high ionisation of knots $\mathrm{L}$ and $\mathrm{T}-\mathrm{V}$, located at the end of the two sections of the jet. In this case a higher ionisation may be produced because the shock propagates into a medium of lower pre-shock density (see Hartigan et al. 1994).

From $n_{\mathrm{e}}$ and $x_{\mathrm{e}}$ we obtain a gross value of the total density of $10^{3}-1.9 \times 10^{4} \mathrm{~cm}^{-3}$. This is maximum for the brightest knots, as expected (the emission in collisionally excited lines is proportional to $\left.n_{\mathrm{e}} \cdot n_{\mathrm{H}}\right)$.

The empty circles in the diagrams of Fig. 3 are the values of $n_{\mathrm{e}}$ and $T_{\mathrm{e}}$ inferred from the [Fe II] lines. From these lines we find a higher electron density, $n_{\mathrm{e}}([\mathrm{Fe} \mathrm{II}]) \sim 1.3-4.3 \times 10^{3} \mathrm{~cm}^{-3}$, and a lower temperature, $T_{\mathrm{e}}([\mathrm{Fe} \mathrm{II}]) \sim 4800-8500 \mathrm{~K}$ than inferred from the optical line ratios. Our result confirms that the observed [Fe II] emission typically comes from regions of the post-shock zone that are more distant from the front than the optical lines, where the gas is cooler and more compressed. Such a situation is described in detail in Nisini et al. (2005). Note also that our values of $n_{\mathrm{e}}$ are lower than those found in Nisini et al. (2002) $\left(n_{\mathrm{e}} \sim 0.5-2 \times 10^{4} \mathrm{~cm}^{-3}\right)$. This is due to the fact that here we use the $[\mathrm{Fe}$ II $] 1.64 \mu \mathrm{m} / 1.32 \mu \mathrm{m}$ ratio to determine extinction, leading us to infer lower values of $A_{\mathrm{V}}$.

Even higher density components can be traced through the $[\mathrm{Fe}$ II $] \lambda 7155 /[\mathrm{Fe}$ II $] \lambda 8617$ and $\mathrm{Ca}$ II $\lambda 8540 /[\mathrm{Ca}$ II $] \lambda 7290$ ratios, as illustrated in Sect. 3.1 (Figs. 4 and 5). From the [Fe II] ratio we infer values of $n_{\mathrm{e}}$ between $\sim 10^{4} \mathrm{~cm}^{-3}$ and $\sim 2.5 \times 10^{5} \mathrm{~cm}^{-3}$ in the closest knots E, F, G2, and G1, that subsequently decreases to $\sim 10^{3} \mathrm{~cm}^{-3}$ in the outer knots $\mathrm{H}, \mathrm{I} 1, \mathrm{~J}$, and L. From the [CaII] ratio we find $n_{\mathrm{e}} \sim 6 \times 10^{5}-2 \times 10^{6} \mathrm{~cm}^{-3}$ in knots E, F, $\mathrm{G} 2, \mathrm{G} 1, \mathrm{H}, \mathrm{J}$, while in the other knots no estimate was possible, because the signal-to-noise is too low. The values of $n_{\mathrm{e}}$ inferred from [Fe II] $\lambda 7155 /[\mathrm{Fe}$ II $] \lambda 8617$ and Ca II $\lambda 8540 /[\mathrm{Ca}$ II $] \lambda 7290$ ratios demonstrate that a component of material even denser than that traced by [Fe II] lines at $1.64,1.60$, and $1.53 \mu \mathrm{m}$ is present in the knots along the jet. If we consider for this higher density component the same ionisation fraction found from the optical lines, one obtains that $n_{\mathrm{H}}$ may be as high as $\sim 10^{7} \mathrm{~cm}^{-3}$ in this layer of the post-shocked regions. Similar high densities have

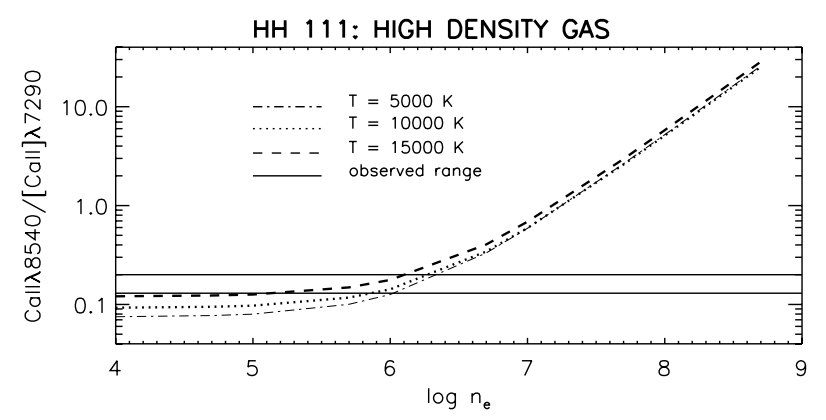

Fig. 5. Comparison between observed and predicted Ca II $\lambda 8540 /[\mathrm{Ca}$ II $] \lambda 7290$ ratios. The solid lines indicate the range of variation of the observed ratio along the jet. The same ratio is computed for three different temperatures with a five-level statistical equilibrium code (dotted, dashed and dash-dotted curves). From this diagnostic, values of the electron density of $\sim 6 \times 10^{5}-2 \times 10^{6} \mathrm{~cm}^{-3}$ are found.

been found in the HH 1 jet (Nisini et al. 2005) and at the base of a few jets from optical T Tauri stars (Hartigan et al. 2004).

Using the $\mathrm{H}_{2}$ lines flux we derived the conditions of the molecular gas in the jet. In the $\mathrm{H}_{2}$ emitting regions the temperature varies between 2000 and $3000 \mathrm{~K}$, and the column density, $N_{\mathrm{H}_{2}}$, between $5 \times 10^{16}$ and $5 \times 10^{17} \mathrm{~cm}^{-2}$. From the latter value divided by the knot size transverse to the line of sight, we derive an approximate $\mathrm{H}_{2}$ density of $\sim 10^{2} \mathrm{~cm}^{-3}$. This is only a lower limit because it is supposed that the $\mathrm{H}_{2}$ lines are excited in a thin layer of molecular material located in the lateral wings of the bow shocks (see above). Thus the dimension of the emitting region is probably smaller than the knot size. Such a picture could be confirmed by high angular resolution images. Nevertheless, the $\mathrm{H}_{2}$ lines trace colder and less dense gas and for this reason the $\mathrm{H}_{2}$ emission is maximum in the outer knots $\mathrm{L}$ and $\mathrm{P}$ (knot $\mathrm{T}-\mathrm{V}$ is out of the frame in the NIR range) as shown by the spatial profiles of Fig. 2.

\section{2. $\mathrm{HH} 34$}

The spectacular HH 34 jet is located in the L1641 cloud in Orion, and is one of the best studied examples of stellar jets. It consists of a parsec-scale flow (Bally \& Devine 1994) the first 30" of which on the blue-shifted side is a well-aligned chain of knots emitting in both optical and NIR lines, placed south of the central source, HH 34 IRS (Eislöffel \& Mundt 1992). HST images of the flow reveal clearly that each knot has the morphology of a mini-bow shock (or "working surface", see, e.g., Ray et al. 1996; Reipurth et al. 2002). Two larger clumpy bow-shocks (HH 34N and $\mathrm{HH} 34 \mathrm{~S}$ ) symmetrically placed at a distance of $\sim 100^{\prime \prime}$ from the source are also visible in the optical (see, e.g., Morse et al. 1992), while the counter-jet is not detected at optical or infrared wavelengths. Proper motions of the knots have been measured from the ground by Eislöffel \& Mundt (1992) and Devine et al. (1997), while Reipurth et al. (2002) made similar measurements with HST deriving typical shock velocities for the working surfaces along the beam of only $20 \mathrm{~km} \mathrm{~s}^{-1}$. Investigations of the physical properties of the jet gas through spectral diagnostic, limited to optical lines, have been carried out by Hartigan et al. (1994) and BE99. In the infrared band, the jet has been investigated by Stapelfeldt et al. (1991), who imaged the jet in [Fe II] lines at moderate and high angular resolution. The only structure previously reported north of the star in the $\mathrm{H}_{2} 2.12 \mu \mathrm{m}$ line, using a narrow-band filter, is an extended arc just south of the optical HH 34N (Stanke et al. 1998). 


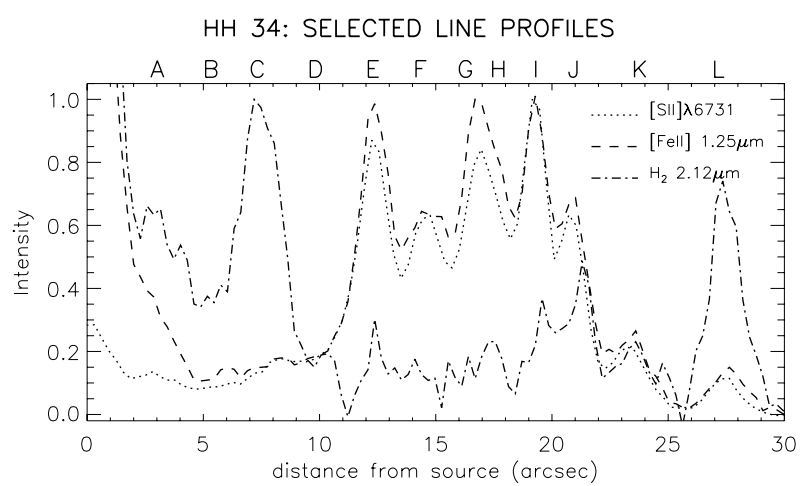

Fig. 6. Same as Fig. 2, but for the HH 34 jet. The zero point of the spatial scale is the source HH 34 IRS (RA(2000): 0535 29.8, $\operatorname{Dec}(2000)$ : -0626 57).

We apply our combined diagnostics to the innermost $30^{\prime \prime}$ of the jet beam where bright optical/NIR lines are seen. We define 12 knots along the jet, following the optical nomenclature of Eislöffel \& Mundt (1992). In Fig. 6 we present the emission profiles in the optical [S II] line, the infrared [Fe II] and $\mathrm{H}_{2}$ lines, each normalized to its intensity peak. The atomic lines are similar both in spatial profile and brightness. The lines intensity is maximum in knot $\mathrm{E}$ (flux $\sim 2 \times 10^{-14} \mathrm{erg} \mathrm{s}^{-1} \mathrm{~cm}^{-2}$ ) and then decreases with distance from the source. The $\mathrm{H}_{2}$ line, on the contrary, is fainter and has a different distribution with two local maxima in knot $\mathrm{C}$ (flux $\sim 2.5 \times 10^{-15} \mathrm{erg} \mathrm{s}^{-1} \mathrm{~cm}^{-2}$ ) and knot L (flux $\sim 1.3 \times 10^{-15} \mathrm{erg} \mathrm{s}^{-1} \mathrm{~cm}^{-2}$ ). As mentioned for HH 111, this behavior is due to the fact that $[\mathrm{S} \mathrm{II}]$ and [Fe II] lines are excited by the J-shock at the apex of the bows, while $\mathrm{H}_{2}$ lines are prevalent in slow, non-dissociative shocks, so the relative spatial distribution of the lines depend on the prevalence of $\mathrm{C}$ or $\mathrm{J}$ shocks along the jet. We note also faint $\mathrm{H}_{2}$ emission between $20^{\prime \prime}$ and $31^{\prime \prime}$, and between $69^{\prime \prime}$ and $80^{\prime \prime}$ north of the star. The latter region corresponds to the arc detected by Stanke et al. (1998). This may indicate the presence of a counterjet that is very extincted with respect to the blue-shifted lobe.

The visual extinction (see Sect. 3.1) is more or less constant in the blue lobe with a value $A_{\mathrm{V}}=1.3 \mathrm{mag}$ in the first $30^{\prime \prime}$ from the source, except for the nearest knot (A), for which we derive $A_{\mathrm{V}} \sim 7.1 \mathrm{mag}$ in agreement with the high extinction value found at the source by Reipurth et al. (1986).

The physical parameters obtained applying the BE technique to the optical lines emitted by each cooling zone traced by the knots are shown in Fig. 7. The inferred values of the electron density averaged over each cooling zone, $n_{\mathrm{e}}$, decrease from a maximum of $3.4 \times 10^{3} \mathrm{~cm}^{-3}$ in the first knot (A) down to $4.3 \times 10^{2} \mathrm{~cm}^{-3}$ in knot D. Then $n_{\mathrm{e}}$ increases again up to a local maximum of $\sim 10^{3} \mathrm{~cm}^{-3}$ in knot $\mathrm{G}$, and from this point on $n_{\mathrm{e}}$ starts to decrease again down to $\sim 0.5-1 \times 10^{2} \mathrm{~cm}^{-3}$ in the outer knots $\mathrm{K}$ and $\mathrm{L}$. The ionisation fraction, $x_{\mathrm{e}}$, is generally quite low in all the knots. It increases from 0.11 in the first knot, A, up to 0.27 in knot B; then decreases gently all along the bright beam down to 0.03 in knots I and J. From there it shows isolated higher values up to 0.08 in knots $\mathrm{K}$ and $\mathrm{L}$. The temperature, $T_{\mathrm{e}}$, derived from the $\mathrm{BE}$ technique, turns out to be on average $\sim 1.4 \times 10^{4} \mathrm{~K}$. From $x_{\mathrm{e}}$ and $n_{\mathrm{e}}$ we obtain an estimate of the total hydrogen density, that shows a trend similar to the electron density. The maximum $n_{\mathrm{H}}$ value is found in knot $\mathrm{A}$ $\left(n_{\mathrm{H}} \sim 3 \times 10^{4} \mathrm{~cm}^{-3}\right)$, then increases from $3 \times 10^{3} \mathrm{~cm}^{-3}$ in knot B up to $2.7 \times 10^{4} \mathrm{~cm}^{-3}$ in knot G. Subsequently it decreases again down to $\sim 10^{3} \mathrm{~cm}^{-3}$ in knots $\mathrm{K}$ and $\mathrm{L}$.
$\mathrm{HH} 34$ DIAGNOSTICS

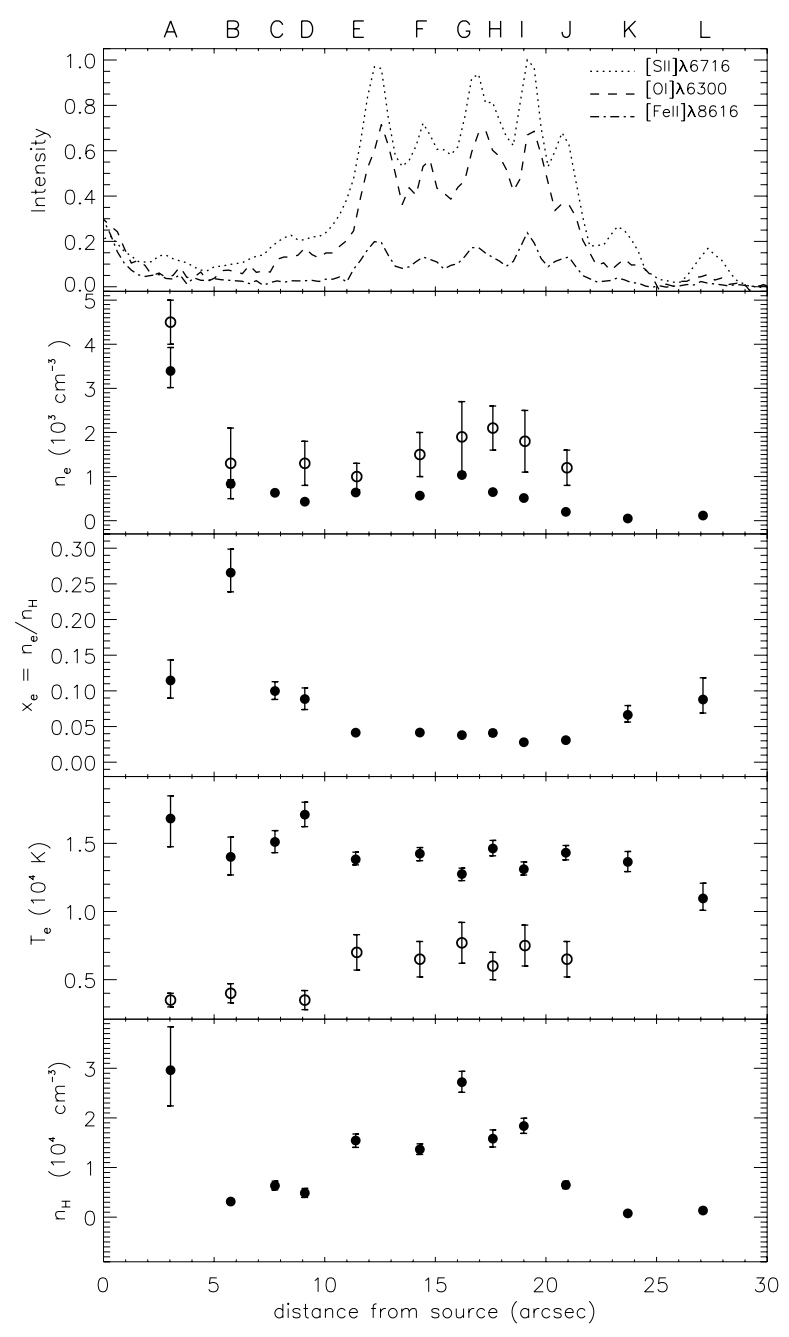

Fig. 7. Same as Fig. 3, but for the HH 34 jet. Open circles are the values derived from [Fe II] line ratios, while filled circles indicate the values determined using the $\mathrm{BE}$ technique. Note that the zero point of the spatial scale is the driving source HH 34 IRS (see Fig. 6).

As noticed for the HH 111 jet in Sect. 3.2, the inferred $x_{\mathrm{e}}$ and $T_{\mathrm{e}}$ values are higher with respect to those obtained for the same object in BE99, due to the differences between the adopted abundances sets. Nevertheless, the trends are the same. The values of $n_{\mathrm{e}}$ and $x_{\mathrm{e}}$ found for HH 34 agree with the electron density derived by Morse et al. (1993), and with the average value of the ionisation $\left(x_{\mathrm{e}}=0.026\right)$ estimated in Hartigan et al. (1994) from the $[\mathrm{N} \mathrm{II}] /[\mathrm{O} \mathrm{I}]$ ratio.

In Fig. 7 we also plot the values of $n_{\mathrm{e}}$ and $T_{\mathrm{e}}$ inferred from the [Fe II] lines. As in the case of $\mathrm{HH} \mathrm{111,} n_{\mathrm{e}}$ derived from the [Fe II] lines is higher than obtained from the [S II] lines $\left(n_{\mathrm{e}} \sim 1-4.5 \times 10^{3} \mathrm{~cm}^{-3}\right)$, while the values for $T_{\mathrm{e}}$ are lower $\left(T_{\mathrm{e}} \sim 3500-7700 \mathrm{~K}\right)$. Such behaviour again corresponds to the expected structure of the shock cooling zones. In this jet too we find an even denser component of gas in the knots, as shown in Figs. 8 and 9 . From the [Fe II] $\lambda 7155 / \lambda 8617$ ratio we infer values from $n_{\mathrm{e}} \sim 4 \times 10^{6} \mathrm{~cm}^{-3}$ close to the source to $n_{\mathrm{e}} \sim 10^{2} \mathrm{~cm}^{-3}$ in knot L. Similar results are obtained through Ca II diagnostics (Fig. 9). In this case we have $n_{\mathrm{e}} \sim 5-6.3 \times 10^{6} \mathrm{~cm}^{-3}$ for the inner knots (from A to D) and $n_{\mathrm{e}}<10^{6} \mathrm{~cm}^{-3}$ in the outer knots (from $\mathrm{E}$ to $\mathrm{L}$ ). Further estimates of relevant physical quantities are discussed in Sect. 5. 
HH 34: HIGH DENSITY GAS

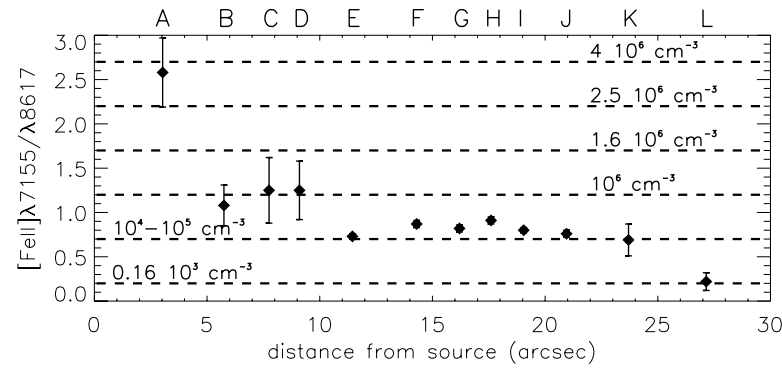

Fig. 8. Same as Fig. 4, but for the HH 34 jet. Values of $n_{\mathrm{e}}$ up to $4 \times$ $10^{6} \mathrm{~cm}^{-3}$ are found. Note that the zero point of the spatial scale is the driving source HH 34 IRS (see Fig. 6).

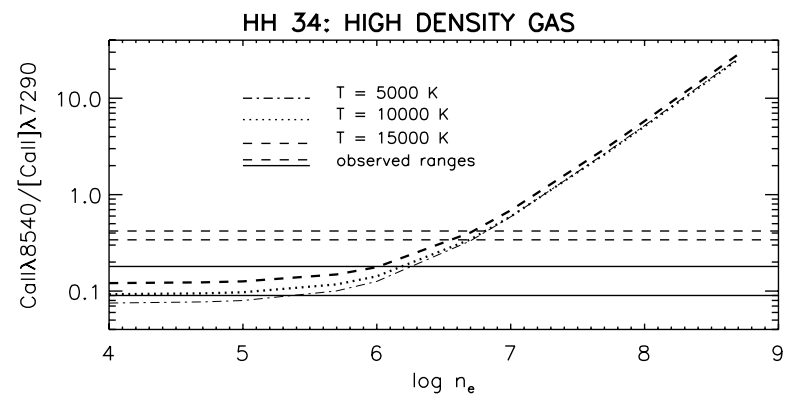

Fig. 9. Same as Fig. 5, but for the HH 34 jet. The horizontal dashed and solid lines indicate the observed range in the knots close to the source (A, B, C , D) and in the outer knots (from E to L), respectively. Electron densities up to $6 \times 10^{6} \mathrm{~cm}^{-3}$ are found in the knots closer to the source.

\section{3. $\mathrm{HH} 83$}

The HH 83 jet is also located in the Orion Nebula, in the L1641 molecular cloud (Reipurth 1989). It is powered by the IRAS source 05311-0631, which is detected at infrared wavelengths only from the $H$ and $K$ band (Moneti \& Reipurth 1995). The jet becomes visible in the optical and near-infrared at a distance of $\sim 10^{\prime \prime}$ from the source, when it emerges from the cloud surface, detected as a reflection nebula $(\operatorname{Re} 17$, Rolph et al. 1990). The jet is composed of 10 blue-shifted knots (following the nomenclature of Reipurth 1989) located between $10^{\prime \prime}$ and $50^{\prime \prime}$ from the source, and terminates with a bow-shock at $\sim 120^{\prime \prime}$. On the opposite side of the source only two faint condensations are found (Reipurth 1989). Because of the faintness of the $\mathrm{H}_{2}$ emission in the acquisition image, the slit was not well aligned for the infrared spectra. This caused the IR spectra to be degraded, and only a few, very faint [Fe II] and $\mathrm{H}_{2}$ lines were observed. Therefore it was not possible to extract the IR spectra for combination with the optical ones. We concentrate on the optical emission from the blue lobe of the jet, applying only the optical diagnostics. From the optical [S II], [O I], and [N II] lines, using the BE technique we derive the values of $n_{\mathrm{e}}, x_{\mathrm{e}}, T_{\mathrm{e}}$, and $n_{\mathrm{H}}$. These are shown in Fig. 10, and are plotted versus the distance from the driving source. The electron density reaches a maximum value of $\sim 0.7 \times 10^{3} \mathrm{~cm}^{-3}$ in knot E; then it decreases with distance from the star down to $\sim 70 \mathrm{~cm}^{-3}$ in knots I-J. The ionisation fraction and the temperature could not be calculated in the first three knots $(\mathrm{A}-\mathrm{B}-\mathrm{C}, \mathrm{D}, \mathrm{E})$ because the diagnostics did not find a solution for the measured line fluxes. This is probably due to the presence of the reflection nebula, the continuum from which could not be properly subtracted, adding spurious flux to the jet emission lines. Concerning the other knots, the ionisation fraction has a maximum value of $\sim 0.55$ in $\mathrm{knot} F$, and
$\mathrm{HH} 83$ DIAGNOSTICS

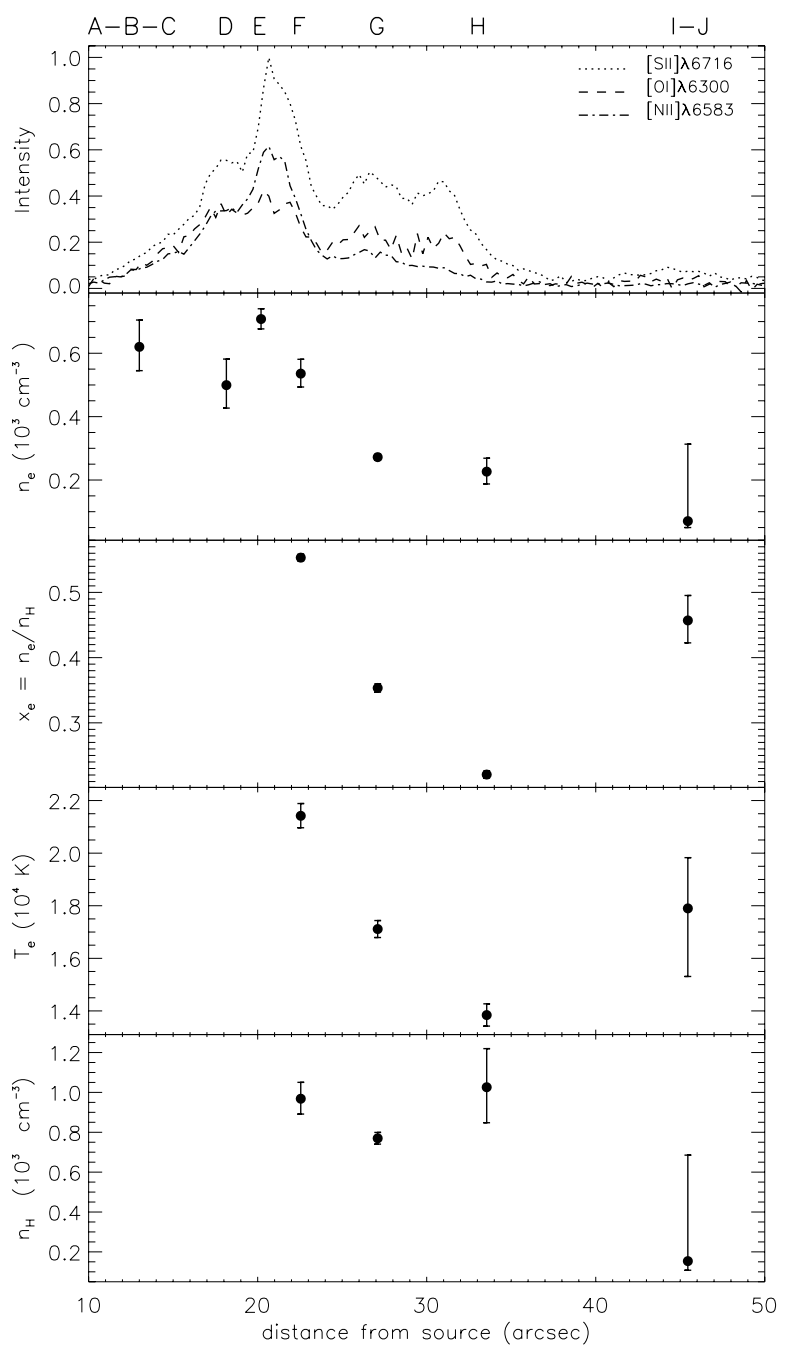

Fig. 10. Same as Fig. 3, but for the HH 83 jet. No infrared diagnostics are available in this case (see text). The zero point of the spatial scale is the source HH 83 IRS (RA(2000): 0533 32.5, Dec(2000): -06 29 44).

then it decreases down to $\sim 0.22$ in knot H. There is another local maximum in knot $\mathrm{I}-\mathrm{J}$, for which we found $x_{\mathrm{e}} \sim 0.46$. The temperature is relatively high, $T_{\mathrm{e}} \sim 2.1 \times 10^{4} \mathrm{~K}$ in knot $\mathrm{F}$ and lower in the following knots, down to $1.4 \times 10^{4} \mathrm{~K}$ in knot $\mathrm{H}$. Then it is higher again in knot $\mathrm{I}-\mathrm{J}\left(T_{\mathrm{e}} \sim 1.8 \times 10^{4} \mathrm{~K}\right)$. Finally, the total density, $n_{\mathrm{H}}$, assumes its highest value of $\sim 10^{3} \mathrm{~cm}^{-3}$ in knots $\mathrm{F}$ and $\mathrm{G}$. We note that a relatively higher degree of ionisation is found for this and the following jets with respect to HH 34 and $\mathrm{HH}$ 111. For an interpretation of this result, as well as for the determination of the mass flux in this flow, see the discussion in Sects. 5.2 and 5.3.

\section{4. $\mathrm{HH} 73$}

The HH 73 jet, discovered by Reipurth \& Graham (1988), is located in the Vela Molecular Ridge $(D=450 \mathrm{pc})$, near the small nebula Re 6 (Reipurth 1981). It is composed of a chain of very faint optical knots, that we name from $A$ to $G$, and extends for a total length of $\sim 70^{\prime \prime}$. Since the source of this jet is not known, the zero point of the spatial scale was set at the beginning of knot A. We do not detect any emission in the near infrared, so we applied our diagnostics only to the optical emission of the 
HH 73 DIAGNOSTICS

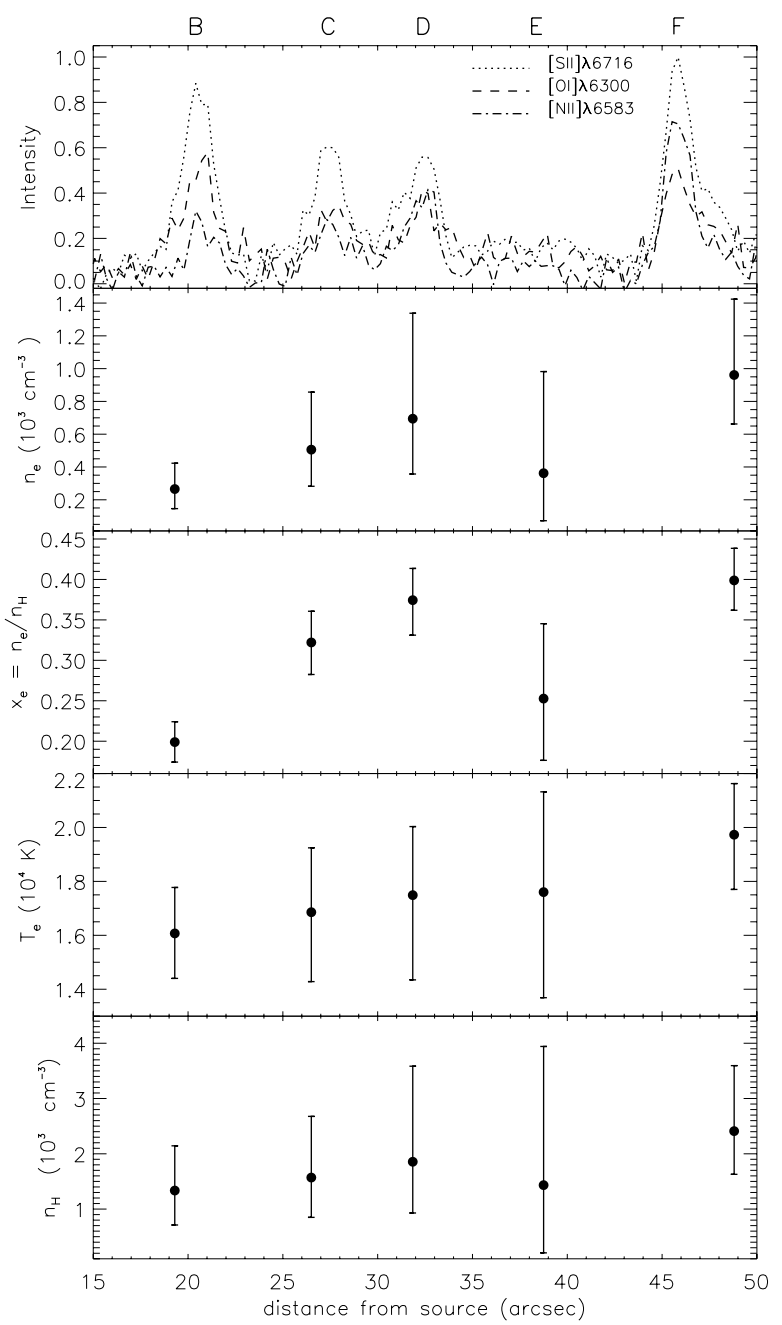

Fig. 11. Same as Fig. 10, but for the HH 73 jet. Due to low signal-tonoise, the results are subject to large errors. Since the source of this jet is not known, the zero point of the spatial scale was set at the beginning of the first detected knot (knot A).

brightest knots (from B to F). The derived values of $n_{\mathrm{e}}, x_{\mathrm{e}}, T_{\mathrm{e}}$, and $n_{\mathrm{H}}$ are shown in Fig. 11. Because of the low signal to noise of the line fluxes, our diagnostics is affected by large errors. Nevertheless, we can define a range of variation for the physical parameters. The values of $n_{\mathrm{e}}$ are between $\sim 0.2 \times 10^{3} \mathrm{~cm}^{-3}$ and $1.4 \times 10^{3} \mathrm{~cm}^{-3}, x_{\mathrm{e}}$ varies between 0.1 and 0.45 , the temperature $T_{\mathrm{e}}$ varies between $\sim 1.4 \times 10^{4}-2.2 \times 10^{4} \mathrm{~K}$, and the total density is in the range of $\sim 1-4 \times 10^{3} \mathrm{~cm}^{-3}$. The relatively high ionisation in this jet may be justified following the same arguments identified for $\mathrm{HH} 83$ (see Sect. 5.3).

\subsection{The HH 24 complex}

The HH 24 complex lies in the NGC 2068 nebula that includes both reflection and emission nebulae, and at least three different outflows (Solf 1987; Mundt et al. 1991; Eislöffel \& Mundt 1997; BE99). IR imaging by Lane (1989) and Zealey et al. (1989) revealed the presence of three optically invisible sources (SSV 63, SSV $63 \mathrm{~W}$, and SSV $63 \mathrm{NE}$ ) which could be the driving sources of the observed outflows. One of the best defined structures is the HH $24 \mathrm{C}$ blue-shifted jet, aligned with the source SSV 63. Eislöffel \& Mundt (1997) showed that the HH 24 C
$\mathrm{HH} 24 \mathrm{C} / \mathrm{E}$ DIAGNOSTICS

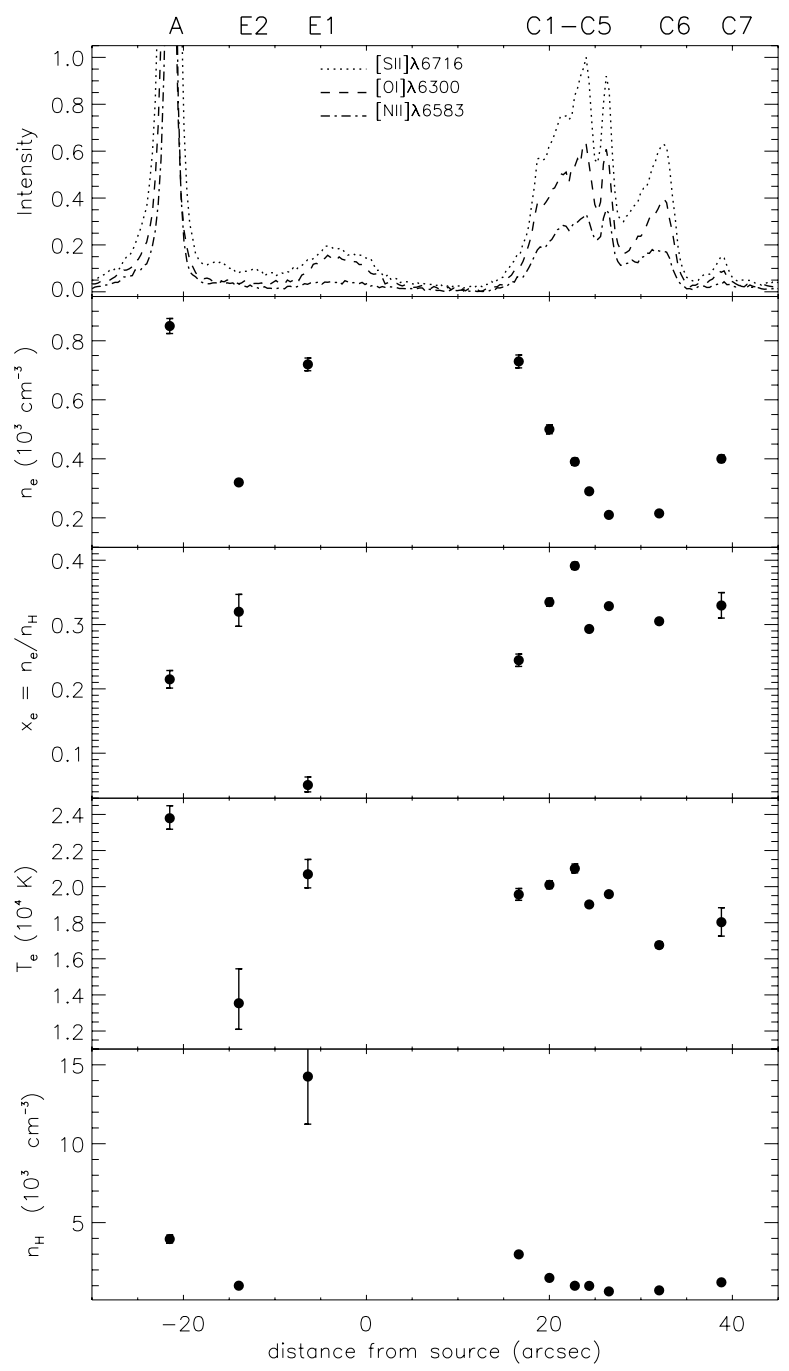

Fig. 12. Same as Fig. 10, but for the $\mathrm{HH} 24 \mathrm{C} / \mathrm{E}$ jet. The zero point of the spatial scale is the source SSV 63, which is supposed to be the driving source of this jet (RA(2000): 0537 08.23, $\operatorname{Dec}(2000)$ : -0009 24.62).

blue lobe extends for $\sim 1 \mathrm{pc}$, including the ring-shaped object called $\mathrm{HH} 24 \mathrm{H}$, and terminating with a giant bow formed by a number of $\mathrm{HH}$ objects (HH 20, HH 21, HH 37, HH 70). On the opposite side there is a chain of red-shifted HH knots (knots of the group $\mathrm{E}$, knot $\mathrm{A}$, and knot $\mathrm{M}$ following the nomenclature of Eislöffel \& Mundt 1997) that seems to form the counterjet, although the structure of this region is still unclear. The red lobe, in fact, deviates from the $\mathrm{HH} 24 \mathrm{C}$ flow axis by $\sim 6^{\circ}$ and there is evidence that the bright knot A may belong to another jet in the complex (HH 24-MMS jet according to Eislöffel $\&$ Mundt 1997). In our spectra we detected the innermost $\sim 80^{\prime \prime}$ of the blue lobe (from knot $\mathrm{C} 1$ to knot C11) and $~ 30^{\prime \prime}$ of the red lobe (knots E1, E2, and A). In the optical many lines are visible throughout all the jet length, while in the infrared we observed only a few faint [Fe II] and $\mathrm{H}_{2}$ lines (from the red-shifted knots E1 and A). As a consequence, our diagnostics is limited to the derivation of the physical parameters in the region of optical emission through the BE technique (see Fig. 12). In the blue lobe the electron density $n_{\mathrm{e}}$ decreases with distance from the source from $\sim 8 \times 10^{2} \mathrm{~cm}^{-3}$ down to $\sim 2 \times 10^{2} \mathrm{~cm}^{-3}$ in knots C5-C6. Then there is a local maximum in knot C7 $\left(n_{\mathrm{e}} \sim 4 \times 10^{2} \mathrm{~cm}^{-3}\right)$. 
As for the red lobe, $n_{\mathrm{e}}$ is equal to $\sim 7 \times 10^{2} \mathrm{~cm}^{-3}$ in knot E1, then it decreases down to $\sim 3 \times 10^{2} \mathrm{~cm}^{-3}$ in knot E2, and finally it reaches a value of $\sim 8.5 \times 10^{2} \mathrm{~cm}^{-3}$ in knot $\mathrm{A}$. The ionisation fraction $x_{\mathrm{e}}$ varies along the jet between $0.2-0.4$, with no definite trend. It is lower in knot E1, where we find $x_{\mathrm{e}} \sim 0.05$. The temperature decreases with distance from the source in the blue lobe going from $\sim 2.1 \times 10^{4} \mathrm{~K}$ down to $\sim 1.7 \times 10^{4} \mathrm{~K}$. In the red lobe, instead, we found a temperature of $\sim 2 \times 10^{4} \mathrm{~K}$ in knot E1, $\sim 1.4 \times 10^{4} \mathrm{~K}$ in knot E2, and a maximum of $\sim 2.4 \times 10^{4} \mathrm{~K}$ in knot A. Finally, the total density is decreasing in the blue lobe from $\sim 3 \times 10^{3} \mathrm{~cm}^{-3}$ in knot $\mathrm{C} 1$ to $6 \times 10^{2} \mathrm{~cm}^{-3}$ in knot $\mathrm{C} 5$, then it increases again in knots C6 and C7 $\left(n_{\mathrm{H}} \sim 0.7-1.2 \times 10^{3} \mathrm{~cm}^{-3}\right)$. Towards the red lobe we find $n_{\mathrm{H}} \sim 1.4 \times 10^{4} \mathrm{~cm}^{-3}$ in knot E1, $n_{\mathrm{H}} \sim 10^{3} \mathrm{~cm}^{-3}$ in knot E2, and $n_{\mathrm{H}} \sim 4 \times 10^{3} \mathrm{~cm}^{-3}$ in knot A. We find higher ionisation fractions and temperatures than those derived by BE99. As mentioned in the case of the HH 34 jet, this is due to the adoption of a different set of abundances (see also Fig. 1). For comments on the comparison with HH 111 and HH 34 see the discussion in Sect. 5.3.

It can be noticed that, while in the blue lobe the derived parameters have a definite trend along the jet, in the red lobe the values are scattered. We find, e.g., that the ionisation fraction and the total density in knot E1 are, respectively, much lower and higher than those found in the $\mathrm{C}$ knots. This effect can be due to the faintness of knots E1 and E2 (see the intensity spatial profiles in Fig. 12) and, as a consequence, to the low signal-to-noise ratio, which affects, overall, the measurement of the $[\mathrm{N} \mathrm{II}] \lambda 6583$ line flux and, thus, the determination of the ionisation fraction. It could also be intrinsic to the object, the structure of which is not entirely clear in this region (Solf 1987; Mundt et al. 1991; Eislöffel \& Mundt 1997) (knot A may not belong to the jet and the emission from the $\mathrm{HH} 24 \mathrm{~J}$ jet can be superimposed on the knots of group E). See further discussion about the HH $24 \mathrm{C} / \mathrm{E}$ jet in Sects. 5.2 and 5.3.

Another jet in the complex is $\mathrm{HH} 24 \mathrm{~J}$. It consists of a chain of knots and condensations, which are well aligned with the source SSV $63 \mathrm{~W}$. This jet also seems to be parsec-scale, terminating with the bow $\mathrm{HH} 19$ to the north-west and the bow-shaped object HH 27 to the south-east (Eislöffel \& Mundt 1997). In the optical we detect emission in the first $\sim 30^{\prime \prime}$ of the north-west lobe and in the first $\sim 40^{\prime \prime}$ of the south-east lobe (HH $24 \mathrm{~J}$ and $\mathrm{HH} 24 \mathrm{~K}$ following the nomenclature of Eislöffel \& Mundt 1997), while no emission lines are detected in the NIR. Unfortunately, in the spectra of the knots, the [S II] $\lambda \lambda 6716,6731$ doublet is not resolved sufficiently as to separately measure the line fluxes, except for two condensations located at $\sim 30^{\prime \prime}$ on the north-west from the source. Since higher resolution spectra of the HH $24 \mathrm{~J}$ jet are not available, we apply our diagnostics only to the two condensations. For these knots we derive an electron density of $\sim 0.5 \times 10^{2} \mathrm{~cm}^{-3}$, a ionisation fraction of $\sim 0.02$ and $\sim 0.05$, a temperature of $\sim 1.5 \times 10^{4} \mathrm{~K}$ and $\sim 0.9 \times 10^{4} \mathrm{~K}$, and a total density of $\sim 2.5 \times 10^{3} \mathrm{~cm}^{-3}$ and $0.9 \times 10^{3} \mathrm{~cm}^{-3}$.

\section{Discussion}

Here we illustrate and discuss further information about the jet physics, derived from the basic gas parameters obtained in Sect. 4 and summarized in Table 2. We also compare the values of these parameters in the various objects, to search for common trends or differences in the sample.

\subsection{Abundances of refractory species}

The wavelength range covered by our spectra includes transitions from refractory species like carbon, calcium and iron. This allows us to check for the gas phase abundances of these species with respect to solar values. In the interstellar medium refractory species are often locked into dust grains, thus a strong depletion is expected. On the other hand, sputtering and photoevaporation processes due to the passage of shock fronts can destroy all or part of dust grains, releasing the refractory atoms into the gas cloud (Jones 2000; Draine 2003). It follows that an estimate of the depletion of the gas phase abundance of these atoms can give important constraints on the dust structure, and on the efficiency of shocks in destroying grains. The latter is related to parameters such as the shock velocity and the pre-shock excitation conditions. In order to infer the elemental gas-phase abundances we compare observed and expected ratios between emission lines of refractory and non-refractory species. We select lines which are excited in the same region of the post-shocked gas, so that one can assume the same filling factor.

For the gas phase abundances of calcium and carbon we use the ratios $[\mathrm{Ca}$ II $] \lambda(7290+7324) /[\mathrm{S} \mathrm{II}] \lambda(6716+6731)$ and $[\mathrm{C} \mathrm{I}] \lambda(9824+9850) /[\mathrm{S} \mathrm{II}] \lambda(6716+6731)$. The predicted values of these ratios are determined using the values of $n_{\mathrm{e}}, x_{\mathrm{e}}$, and $T_{\mathrm{e}}$ inferred through the BE technique. Calcium is assumed to be completely ionised while the carbon ionisation fraction is computed considering collisional and charge exchange ionisation and direct and dielectronic recombination processes (rates from Stancil et al. 1998; and Landini \& Monsignori Fossi 1990). Finally, we assumed solar abundances from Asplund et al. (2005) for $\mathrm{Ca}$ and C. In Fig. 13 the comparison between predicted and observed ratios along the $\mathrm{HH} 111$ and $\mathrm{HH} 34$ jets is shown.

The observed [Ca II $] \lambda(7290+7324) /[\mathrm{S} \mathrm{II}] \lambda(6716+6731)$ ratio is smaller than the predicted one along both HH 34 and HH 111. We interpret this discrepancy as due to depletion of Calcium atoms with respect to the solar value (see Table 3). Along the HH 111 jet a depletion of $\sim 50 \%$ is found in the knots closest to the source. Then the depletion slowly decreases with distance, down to zero in knot $\mathrm{O}$. Another local maximum is then found in knot P. Similarly, along HH 34 we find a maximum depletion $(\sim 70 \%)$ in the first knot, which then decreases steadily until a solar gas-phase abundance is reached in the outermost three knots $(\mathrm{J}, \mathrm{K}, \mathrm{L})$.

The trend, observed along both HH 111 and HH 34, can be explained by the fact that the leading bow-shock has a much larger effective shock velocity (of $100-400 \mathrm{~km} \mathrm{~s}^{-1}$ ) than the internal working surfaces in the beam $\left(30-40 \mathrm{~km} \mathrm{~s}^{-1}\right)$ (Raga \& Kofman 1992). On the other hand, the shock models predict substantial grain destruction only for shock speeds $>100 \mathrm{~km} \mathrm{~s}^{-1}$ (Draine 2003). We would therefore only expect the dust to be gradually destroyed by the working surface as it propagate outwards. At leading bow-shocks however, e.g. HH 34 S, almost total destruction should be achieved. Note that along the jet we measure less depletion than in the surrounding ambient medium (see, e.g., Baldwin et al. 1991), which is another indication that dust is partially destroyed by jet shocks.

For carbon, instead, good agreement between the observed and predicted $[\mathrm{CI}] \lambda(9824+9850) /[\mathrm{SII}] \lambda(6716+6731)$ ratio is found, at least in the brightest knots where the signal-to-noise is high. This indicates that this species is not depleted in the Orion Cloud, a result that has also been found in previous works (e.g., Rubin et al. 1991; Baldwin et al. 1991; Peimbert et al. 1993; Esteban et al. 2004). 
Table 3. Gas-phase abundance of refractory species with respect to the solar abundances determined by Asplund et al. (2005).

\begin{tabular}{c|ccc|cc}
\hline \hline Species & \multicolumn{3}{|c}{$[\mathrm{X}]_{\text {gas }} /[\mathrm{X}]_{\text {solar }}$} & \multicolumn{2}{c}{ Abundances: $(\mathrm{X} / \mathrm{H})$} \\
& HH 111 & HH 34 & Orion Cloud & Orion abundances $^{a}$ & Solar abundances $^{b}$ \\
\hline $\mathrm{Ca}$ & $0.5-1$ & $0.3-1$ & 0.01 & $2 \times 10^{-8}$ & $2.04 \times 10^{-6}$ \\
$\mathrm{C}$ & $\sim 1$ & $\sim 1$ & 1.07 & $2.63 \times 10^{-4}$ & $2.45 \times 10^{-4}$ \\
$\mathrm{Fe}$ & - & 0.13 & 0.05 & $1.29 \times 10^{-6}$ & $2.82 \times 10^{-5}$ \\
\hline
\end{tabular}

${ }^{a}$ from Esteban et al. (2004), except for the Ca abundance which was determined by Baldwin et al. (1991); ${ }^{b}$ from Asplund et al. (2005).

\section{Ca and C DEPLETION}
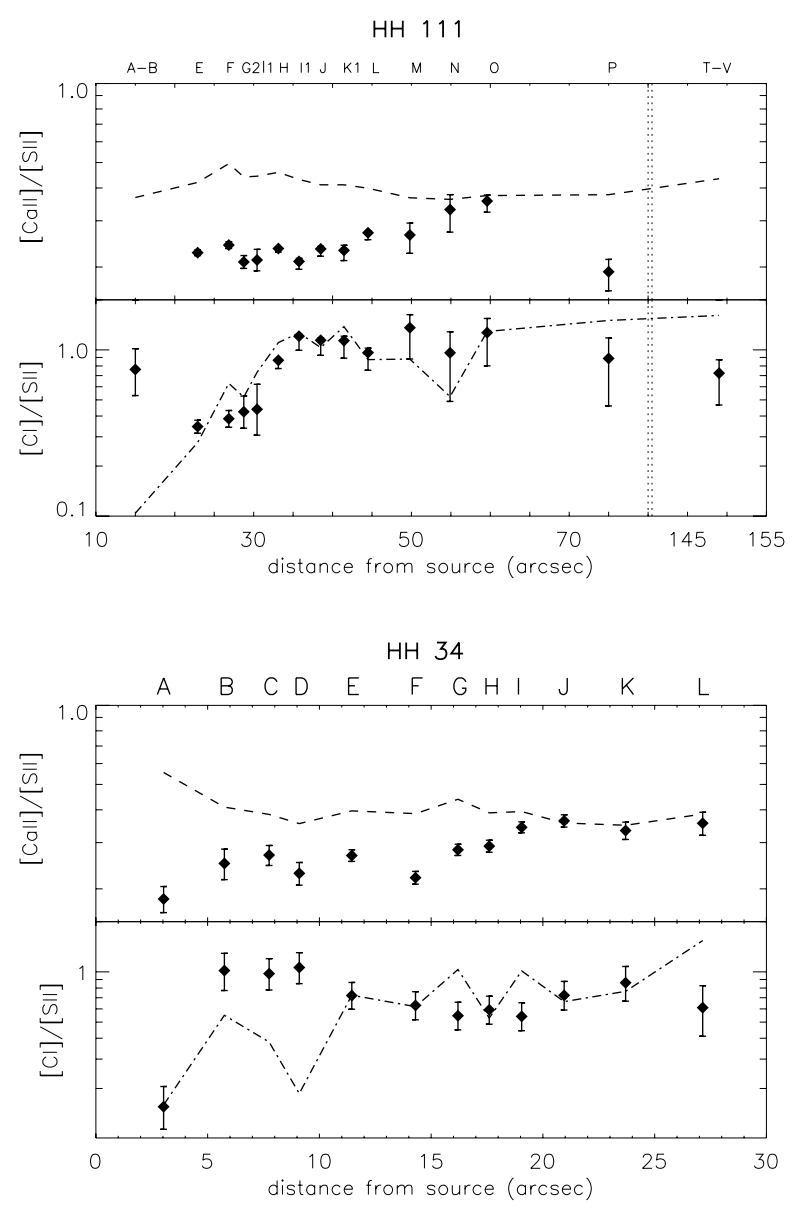

Fig. 13. Comparison between observed (diamonds) and predicted (dashed and dash-dotted lines) $[\mathrm{Ca}$ II $] \lambda(7290+7324) /[\mathrm{S} \mathrm{II}] \lambda(6716+$ $6731)$ and $[\mathrm{CI}] \lambda(9824+9850) /[\mathrm{S} \mathrm{II}] \lambda(6716+6731)$ ratios along the $\mathrm{HH} 111$ and $\mathrm{HH} 34$ jets. The theoretical ratios are computed from the derived physical parameters of the gas and assuming solar abundances for $\mathrm{Ca}$ and $\mathrm{C}$. The $[\mathrm{Ca} \mathrm{II}] /[\mathrm{S} \mathrm{II}]$ ratios indicate a depletion of the gas phase abundance of $\mathrm{Ca}$ of $\sim 50 \%$ in the innermost knots. The depletion decreases with distance from the source for both $\mathrm{HH} 111$ and $\mathrm{HH} 34$. The good agreement between the observed and theoretical $[\mathrm{CI}] /[\mathrm{S} \mathrm{II}]$ ratios indicates that carbon is not depleted with respect to solar values. Note the vertical broken lines in the HH 111 plot indicate a gap in the distance axis.

In a similar way we determine the quantity of Iron atoms in gaseous form. For Fe the comparison is complicated by the fact that the observed [Fe II] lines come from regions of the post-shocked gas different from those giving rise to the optical lines. This is why contrasting results are found in the literature, in works that adopt different methods (see e.g. Beck-Winchatz et al. 1996; Böhm et al. 2001; Nisini et al. 2002). Since we

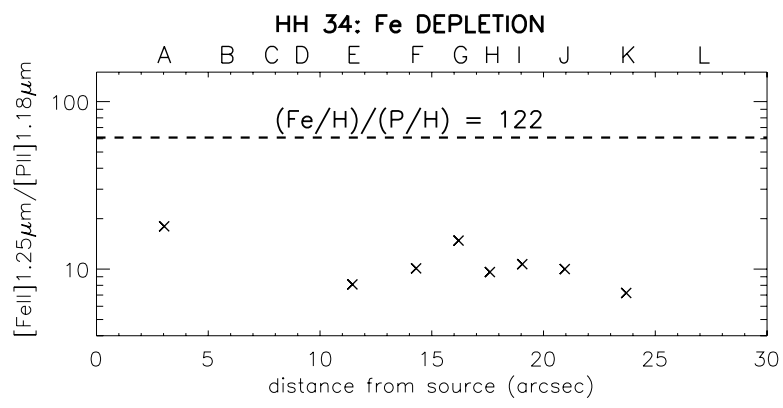

Fig. 14. Comparison between the observed [Fe II] $1.25 \mu \mathrm{m} /[\mathrm{P}$ II $] 1.18 \mu \mathrm{m}$ ratios (crosses) and the expected value obtained assuming solar abundances for Fe and P (Oliva et al. 2001). The position of the observed points indicates a depletion of $\sim 85 \%$ of the gas phase abundance of Iron.

obtained different values of $n_{\mathrm{e}}$ and $T_{\mathrm{e}}$ from [Fe II] and [S II] lines, we cannot compare ratios between these lines, because they have probably different filling factors (see Nisini et al. 2005). We use, instead, the ratio [Fe II] $1.25 \mu \mathrm{m} /[\mathrm{P}$ II $] 1.18 \mu \mathrm{m}$. Phosphorus, in fact, is a non-refractory species, and the excitation conditions of the $1.18 \mu \mathrm{m}$ line are similar to the ones of the $[\mathrm{Fe}$ II $] 1.25 \mu \mathrm{m}$ line. The predicted [Fe II] $1.25 \mu \mathrm{m} /[\mathrm{P}$ II $] 1.18 \mu \mathrm{m}$ is estimated to be about $[(\mathrm{Fe} / \mathrm{H}) /(\mathrm{P} / \mathrm{H})] / 2$ (Oliva et al. 2001) if Iron is not depleted. The comparison between the observed ratio in the HH 34 jet and the one calculated assuming solar abundances is shown in Fig. 14. From the figure we deduce that in $\mathrm{HH} 34 \mathrm{Fe}$ may be depleted by $87 \%$ with respect to solar $\left((\mathrm{Fe} / \mathrm{H})_{\text {gas }} /(\mathrm{Fe} / \mathrm{H})_{\text {solar }} \sim 0.13\right.$ and thus $\left.(\mathrm{Fe} / \mathrm{H})_{\text {gas }} \sim 3.67 \times 10^{-6}\right)$. This depletion is lower than the one found by Esteban et al. (2004) in the ISM of Orion, so some atoms of Iron have probably been unlocked by the jet shocks. The fact that we found a different amount of depletion for Calcium and Iron indicates that the different species follow selective patterns for their erosion from dust grains as expected from theory (Jones 2000). Similar results have been also obtained on the HH 1 jet in Nisini et al. (2005) indicating a common behavior of the erosion patterns in different jets. We could not check the iron depletion along the HH 111 jet, since the $[\mathrm{P}$ II $] 1.18 \mu \mathrm{m}$ line is not visible in our spectra.

\subsection{Determination of the mass flux rate and other jet parameters}

The mass flux rate $\left(\dot{M}_{\text {jet }}\right)$ is a fundamental quantity governing the jet dynamics and which enters all comparisons between observations and theoretical models. For example, in the magnetohydro dynamic models proposed to explain jet formation and acceleration (see Königl \& Pudritz 2000; Shu et al. 2000), the ratio between the rate of mass ejected into the jet $\left(\dot{M}_{\text {jet }}\right)$ and the rate of mass accreted from the disk onto the star $\left(\dot{M}_{\text {acc }}\right)$ is fixed $\left(\dot{M}_{\text {jet }} / \dot{M}_{\text {acc }} \sim 0.01-0.1\right)$. Moreover, the knowledge of $\dot{M}_{\text {jet }}$ allows us to estimate other important dynamical quantities such 
as the linear $\left(\dot{P}_{\text {jet }}\right)$ and angular $\left(\dot{L}_{\text {jet }}\right)$ momentum fluxes carried by the jet. Knowing $\dot{P}_{\text {jet }}$ one can check if the jet is powerful enough to accelerate surrounding molecular outflows, thus helping to clear the circumstellar environment and inject turbulence into the cloud. $\dot{L}_{\text {jet }}$ is related instead to the jet's capability of removing excess angular momentum from the disk/star system, thus allowing the accretion of matter from the disk onto the central star (Königl \& Pudritz 2000; Woitas et al. 2005).

The determination of the physical conditions along the jet allows us to derive $\dot{M}_{\text {jet }}$ in each knot. We use two different procedures, as we also did in Nisini et al. (2005) for the HH 1 jet. In the first method $(\operatorname{method} \mathrm{A})$, we estimate the mass flux as $\dot{M}_{\text {jet }}=\mu m_{\mathrm{H}} n_{\mathrm{H}} \pi r_{\mathrm{J}}^{2} v_{\mathrm{J}}$, where $\mu=1.24$ is the mean atomic weight, $m_{\mathrm{H}}$ the proton mass, $n_{\mathrm{H}}$ the hydrogen density and $r_{\mathrm{J}}$ and $v_{\mathrm{J}}$, respectively, the jet radius and velocity. We use the total density inferred from our diagnostics and the values of $r_{\mathrm{J}}$ and $v_{\mathrm{J}}$ taken from the literature, as our spectral resolution is too low for such an estimate ( $r_{\mathrm{J}}$ is taken to be one half of the FWHM of the [S II] intensity profile in HST images). This method is independent of the reddening estimate, but it assumes that the knot is uniformly filled at the density derived from the diagnostic, giving an upper limit to $\dot{M}_{\text {jet }}$. Such an effect is partially compensated for by the presence of regions at even higher densities in the beams than those traced by the [S II] lines, which are not taken into account in the calculations. Alternatively (method B), $\dot{M}_{\text {jet }}$ can be derived from the observed luminosity, L(line), of selected optically thin lines such as $[\mathrm{S} \mathrm{II}],[\mathrm{OI}]$, and [Fe II], that is proportional to the mass of the emitting gas: $\dot{M}_{\text {jet }}=\mu m_{\mathrm{H}}\left(n_{\mathrm{H}} V\right) v_{\mathrm{t}} / l_{\mathrm{t}}$, with $n_{\mathrm{H}} V=L($ line $)\left(h v A_{\mathrm{i}} f_{\mathrm{i}} \frac{X^{\mathrm{i}}}{X} \frac{X}{H}\right)^{-1}$, where $V$ is the volume effectively filled by the emitting gas, $v_{\mathrm{t}}$ and $l_{\mathrm{t}}$ are the tangential velocity and length of the knot, $A_{\mathrm{i}}$ and $f_{\mathrm{i}}$ the radiative rate and the upper level population relative to the considered transition, $\frac{X^{\mathrm{i}}}{X}$ and $\frac{X}{H}$ are the ionisation fraction and the relative abundance of the considered species. This method is affected by uncertainties in absolute calibrations, extinction, and distance, but does implicitly take into account the volume filling factor $f f$, which, in practice, is simply the ratio of $\dot{M}_{\text {jet }}$ values derived from methods B and A. In fact, the method only measures gas which is sufficiently heated to radiate the observed lines.

The mass fluxes inferred for the HH 111, HH 34, and HH 83 jets are shown in Fig. 15. For HH 111, method A gives a mass flux approximately constant with distance from the source and equal to about $\sim 2 \times 10^{-7} M_{\odot} \mathrm{yr}^{-1}\left(r_{\mathrm{J}}\right.$ are from Reipurth et al. 2000; and $v_{\mathrm{t}}$ from Hartigan et al. 2001). Method B gives smaller values of $\dot{M}_{\text {jet }}$, that, however, decrease with distance from the source. A steady jet flow is actually expected to show a more or less constant $\dot{M}_{\text {jet }}$ along the beam (as derived from method A) unless significant mass is loss sideways by, e.g., a turbulent boundary layer or big bow shocks. This seems to be the case for $\mathrm{HH}$ 111. A possible explanation for the observed decrease is that the jet opening angle increases with distance from the source to the point that the jet diameter becomes larger than the slit width from $30^{\prime \prime}$ outwards. Thus method B determinations are affected by partial loss of line flux. To obtain a value of the mass flux which takes into account the beam filling, we use the filling factor from the knots in the first $30^{\prime \prime}(f f \sim 0.2)$ to correct the values obtained from method A. We obtain an average $\dot{M}_{\text {jet }}$ value of $\sim 5 \times 10^{-8} M_{\odot} \mathrm{yr}^{-1}$. Finally, we estimate the mass flux transported by the molecular component through the inferred $\mathrm{H}_{2}$ column density and the velocity of the molecular gas measured by Davis et al. (2001). From method A we obtain $\dot{M}_{\text {jet }}\left(\mathrm{H}_{2}\right) \sim 10^{-9}-10^{-10} M_{\odot} \mathrm{yr}^{-1}$, that is down two orders of magnitude with respect to the atomic component. As explained in the
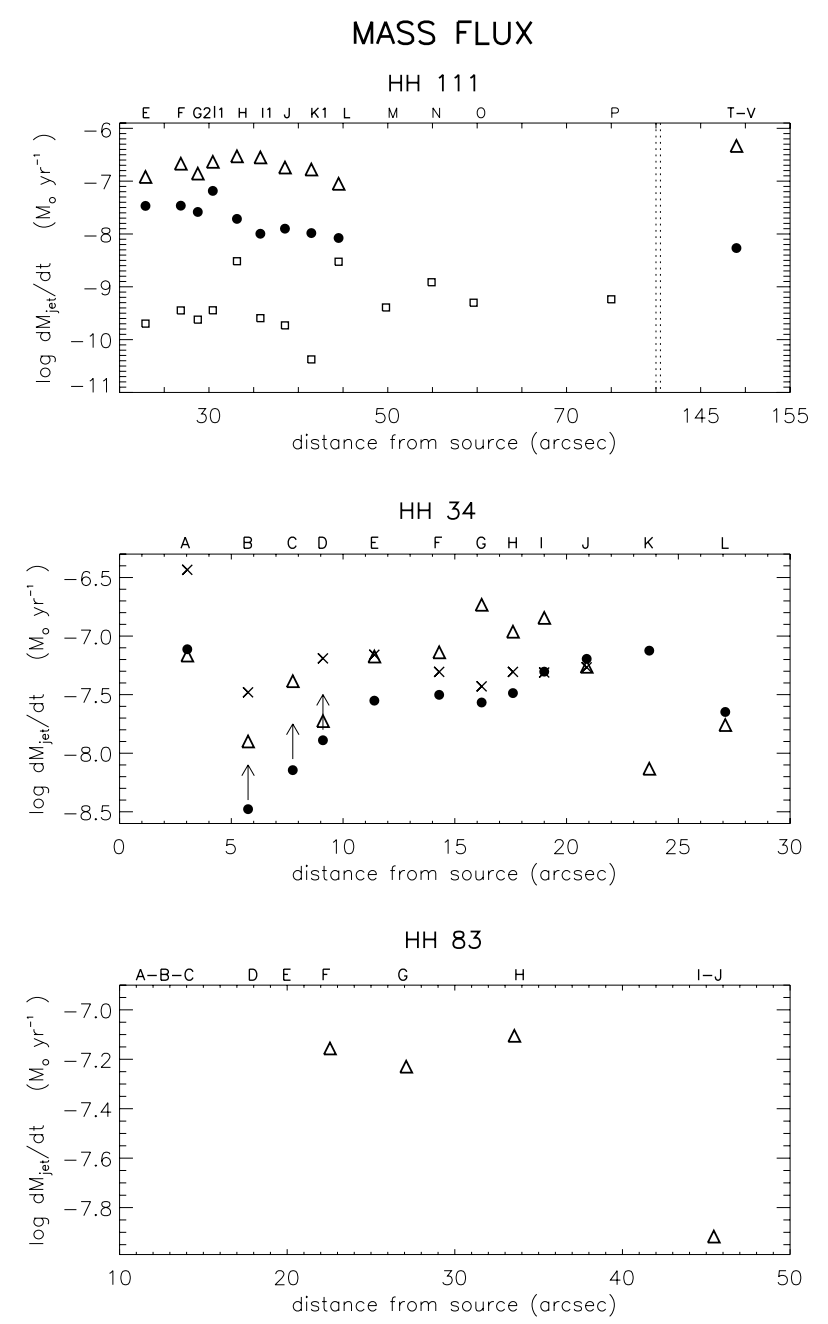

Fig. 15. Mass fluxes determined for the HH 111, HH 34, and HH 83 jets. Triangles refer to values of $\dot{M}_{\text {jet }}$ inferred from our estimates of the total density, $n_{\mathrm{H}}$, and the values of the jet radius $\left(r_{\mathrm{J}}\right)$ and velocity $\left(v_{\mathrm{J}}\right)$ taken from the literature (method A). Circles $\left(\mathrm{S}^{+}, \mathrm{N}^{+}, \mathrm{O}^{0}\right)$ and crosses ([Fe II]) refer to the estimates of $\dot{M}_{\text {jet }}$ derived from line luminosities (method B). This method takes into account the knot beam filling. Squares refer to the values of the mass flux transported by the molecular component. These values are calculated using our estimates of $\mathrm{H}_{2}$ column density $(\operatorname{method} \mathrm{A})$.

previous section, $\mathrm{H}_{2}$ emission is thought to arise in the $\mathrm{C}$-shocks that form in the lateral wings of the bow shocks. Although high angular resolution images in these lines are not yet available to confirm this picture, the low value we find for the mass loss rate is consistent with $\mathrm{H}_{2}$ tracing only a thin shocked layer of the jet.

For HH 34 we obtain again different values from methods A and $\mathrm{B},\left(r_{\mathrm{J}}\right.$ and $v_{\mathrm{t}}$ are from Reipurth et al. 2002) but in this case both are constant with distance, as the jet beam is always smaller than the slit width. Note that the values of $\dot{M}_{\text {jet }}$ derived from [Fe II] luminosity are a lower limit since in this calculation we assumed that all $\mathrm{Fe}$ is in gaseous form. Taking into account the beam filling, and considering only the brightest knots $(E-J)$ for which we have good signal-to-noise, we infer an average value of the mass flux of $\sim 3.9 \times 10^{-8} M_{\odot} \mathrm{yr}^{-1}$. It can be noticed that both for $\mathrm{HH} 111$ and $\mathrm{HH} 34$ the values of $\dot{M}_{\text {jet }}$ derived from method A are similar to the ones found by Hartigan et al. (1994) using the same technique. The values obtained taking into account the volume filling factor (method B) are in both cases lower by one order of magnitude. The latter values are a better 
Table 4. Mass flux and linear momentum flux along the jets.

\begin{tabular}{c|ccc|cccc|c}
\hline \hline Jet & $\begin{array}{c}v_{\text {jet }}{ }^{a} \\
\left(\mathrm{~km} \mathrm{~s}^{-1}\right)\end{array}$ & $\begin{array}{c}r_{\text {jet }}{ }^{b} \\
\left({ }^{\prime \prime}\right)\end{array}$ & $\begin{array}{c}n_{\mathrm{H}}{ }^{c} \\
\left(10^{3} \mathrm{~cm}^{-3}\right)\end{array}$ & $f f^{d}$ & $\begin{array}{c}\dot{M}_{\text {jet }}(\mathrm{A})^{e} \\
\left(M_{\odot} \mathrm{yr}^{-1}\right)\end{array}$ & $\begin{array}{c}\dot{M}_{\text {jet }}(\mathrm{B})^{f} \\
\left(M_{\odot} \mathrm{yr}^{-1}\right)\end{array}$ & $\begin{array}{c}\dot{P}_{\text {jet }}{ }^{g} \\
\left(M_{\odot} \mathrm{yr}^{-1} \mathrm{~km} \mathrm{~s}^{-1}\right)\end{array}$ & $\begin{array}{c}\dot{P}_{\text {outflow }}{ }^{h} \\
\left(M_{\odot} \mathrm{yr}^{-1} \mathrm{~km} \mathrm{~s}^{-1}\right)\end{array}$ \\
\hline HH 111 & 268 & $0.25-1.0$ & 11.3 & 0.2 & $2.2 \times 10^{-7}$ & $5.0 \times 10^{-8}$ & $1.3 \times 10^{-5}$ & $1.8 \times 10^{-5}$ \\
HH 34 & 211 & $0.15-0.35$ & 16.2 & 0.4 & $1.1 \times 10^{-7}$ & $3.9 \times 10^{-8}$ & $0.8 \times 10^{-5}$ & $1.2 \times 10^{-6}$ \\
HH 83 & 213 & 0.8 & 0.9 & - & $6.9 \times 10^{-8}$ & - & $1.5 \times 10^{-5}$ & - \\
HH 24 C & 425 & 0.6 & 1.3 & - & $9.9 \times 10^{-8}$ & - & $4.2 \times 10^{-5}$ & - \\
\hline
\end{tabular}

${ }^{a} v_{\text {jet }}$ is derived from $v_{\mathrm{t}}$ and $v_{\mathrm{r}}$ estimated by: Hartigan et al. (2001, HH 111), Reipurth et al. (2002, HH 34), Reipurth (1989, HH 83) and Mundt et al. (1991, HH 24 C). ${ }^{b} r_{\text {jet }}$ is taken to be one half of the FWHM of the [S II] intensity profile measured by Reipurth et al. (2000, HH 111), Reipurth et al. (2002, HH 34), Mundt et al. (1991, HH 83, HH 24 C). ${ }^{c} n_{\mathrm{H}}$ is the total density estimated via the BE technique (see Sect. 3.2). ${ }^{d} f f$ is the volume filling factor estimated from the ratio between observed and theoretical line luminosities; this is equal to the ratio of $\dot{M}_{\text {jet }}$ values derived from methods B and A (see text). ${ }^{e} \dot{M}_{\text {jet }}$ estimated through method A (see text) and averaged over the brightest knots; a $f f=1$ is assumed. ${ }^{f} \dot{M}_{\text {jet }}$ measured from [S II] and [OI] line luminosities taking into account the beam filling (method B) and averaged over the brightest knots. ${ }^{g} \dot{P}_{\text {jet }}$ is calculated from $\dot{M}_{\text {jet }}(\mathrm{B})$ and $v_{\text {jet }}\left(\dot{P}_{\text {jet }}=\dot{M}_{\text {jet }} v_{\text {jet }}\right) .{ }^{h} \dot{P}_{\text {outflow }}$ is the flux of linear momentum transported by the molecular CO flows and measured by Chernin \& Masson (1995, HH 34) and Reipurth \& Olberg (1991; Cernicharo \& Reipurth 1996, HH 111).

estimate of the mass flux transported by the atomic optical component. Nevertheless, they are lower limits to the total mass flux since we are not considering even denser components nor the material that does not radiate (as shown, e.g., by the $\dot{M}_{\text {jet }}([\mathrm{Fe}$ II $]$ ) estimates).

For HH 83 we infer the mass flux from the derived total density and the values of the jet radius and velocity from Mundt et al. (1991) and Reipurth (1989). We could not estimate the mass flux from line luminosities because we do not have a measure of the visual extinction, due to the non-detection of NIR lines. The mass flux from method A is $\dot{M}_{\text {jet }} \sim 6.9 \times 10^{-8} M_{\odot} \mathrm{yr}^{-1}$. Lacking an estimate of $f f$ here, this is only an upper limit.

For $\mathrm{HH} 73$, and $\mathrm{HH} 24 \mathrm{~J}$, no literature determinations of the jet radius, velocity, and the angle of inclination with respect to the plane of the sky are available. Thus we could not estimate the mass flux for these objects.

Finally, for $\mathrm{HH} 24 \mathrm{C} / \mathrm{E}$ we obtain raw estimates of the average mass flux from the average jet radius and radial velocity given by Mundt et al. (1991). Combining our average value of the total density and assuming an inclination angle with respect to the plane of the sky of $25^{\circ}$ (BE99) we find a mass flux of $\sim 9.9 \times 10^{-8} M_{\odot} \mathrm{yr}^{-1}$.

For our sample of $\mathrm{HH}$ jets we determine the linear momentum as $\dot{P}_{\text {jet }}=\dot{M}_{\text {jet }} v_{\text {jet }}$, combining the estimated $\dot{M}_{\text {jet }}$ with the average values of $v_{\mathrm{J}}$ taken from the literature. We find $\dot{P}_{\text {jet }} \sim 1.3 \times 10^{-5} M_{\odot} \mathrm{yr}^{-1} \mathrm{~km} \mathrm{~s}^{-1}$ for $\mathrm{HH} 111, \dot{P}_{\text {jet }} \sim 0.8 \times$ $10^{-5} M_{\odot} \mathrm{yr}^{-1} \mathrm{~km} \mathrm{~s}^{-1}$ for $\mathrm{HH} 34, \dot{P}_{\text {jet }} \sim 1.5 \times 10^{-5} M_{\odot} \mathrm{yr}^{-1} \mathrm{~km} \mathrm{~s}^{-1}$ for $\mathrm{HH} 83$, and $\dot{P}_{\text {jet }} \sim 4.2 \times 10^{-5} M_{\odot} \mathrm{yr}^{-1} \mathrm{~km} \mathrm{~s}^{-1}$ for HH $24 \mathrm{C}$. The results are summarized in Table 4.

For HH 111 and HH 34 we compare these values with those measured for the molecular outflows seen in CO lines. These were estimated by Chernin \& Masson (1995) for HH 34 and Reipurth \& Olberg (1991) and Cernicharo \& Reipurth (1996) for HH 111. The comparison (see Table 4) shows that the flux of linear momentum carried by the $\mathrm{HH} 111$ and $\mathrm{HH} 34$ jets is higher or comparable to that of the molecular outflows. Thus in principle the jets appear to be capable of accelerating these outflows.

In order to measure the flux of angular momentum carried away by the jet, we need measurements of the jet toroidal velocity, which requires sub-arcsecond resolution spectra (see, e.g., Bacciotti et al. 2002; Coffey et al. 2004; Woitas et al. 2005). To this aim one should acquire high angular and spectral resolution spectra with ground-based instruments equipped with adaptive optics.

\subsection{Comparison between the examined objects}

Our analysis allows us to compare physical parameters of the jets in our sample. In particular, it is interesting to investigate whether the various excitation conditions in our sample arise from different characteristics intrinsic to the jets, or are set by the environment through which the jets propagate. From the analysis of the reduced spectra we can divide the examined targets into two classes: (I) jets visible both in the optical and in the nearinfrared (i.e. HH 111 and HH 34) and (II) jets that show few, very faint or no lines in the near-infrared (i.e. HH 83, HH 73 and HH $24 \mathrm{C} / \mathrm{E}$ ). The parameters collected in Table 2 show that the jets which are not visible or faint in the infrared are less dense than those that show NIR emission. This is expected, since the $\mathrm{Fe}^{+}$levels from which infrared lines originate, have critical densities $>10^{4} \mathrm{~cm}^{-3}$. Thus, electron densities $>10^{3} \mathrm{~cm}^{-3}$ are needed to populate them. Jets in group (II) also show higher ionisation fractions and temperatures. The fact that the ionisation fractions are higher in the less dense jets is consistent with the shocks propagating in a medium of low preshock density. This produces a higher excitation for a given shock velocity (Hartigan et al. 1994). The low electron density also slows down the recombination process, keeping the level of ionisation higher. An external source of UV radiation could also contribute to the excitation conditions. Such a source, however, remains unidentified.

The above arguments would indicate that the ionizing agent is identical and has the same efficiency, in both groups of jets. On the other hand, the lower temperatures inferred for Group (I) jets indicate that the cooling process is more efficient in the denser objects, as expected for collisional line excitation.

We also note that the mass flux does not vary significantly for the two samples. If we expect a steap decrease of the mass flux with age, it seems that the sources of the two different groups have similar ages.

$\mathrm{HH} 111$ and $\mathrm{HH} 34$ are two examples of giant $\mathrm{HH}$ flows and have similar characteristics such as: (i) a well defined blue lobe consisting of a long chain of bright knots and an almost completely obscured red lobe; (ii) the presence of distant bowshocks, such as the bow $\mathrm{T}-\mathrm{V}$ along the $\mathrm{HH} 111$ blue lobe at a distance of $150^{\prime \prime}$ from the source and the HH $34 \mathrm{~N}$ and HH $34 \mathrm{~S}$ bows at a distance of $100^{\prime \prime}$ from HH 34 IRS (Reipurth \& Bally 2001); (iii) similar velocities (see Table 4); (iv) similar [S II] and [Fe II] profiles decreasing with distance from the source; (v) similar physical gas conditions (see Table 2); (vi) both jets have a denser component traced by [CaII] and [Fe II] lines; (vii) along both jets carbon is of solar abundance, while 
calcium is depleted. Moreover the depletion has a similar trend, decreasing with the distance from the source; (viii) similar flux of mass and linear momentum (see Table 4). On the other hand, they show important differences. The HH 34 jet is less extincted and both the source and the inner knots are visible in the optical, while the HH 111 source is deeply embedded in the parental cloud and the jet becomes visible only at a distance of $\sim 15^{\prime \prime}$ from the source. As a consequence the HH 34 jet is associated with a small molecular outflow, while along the HH 111 axis a powerful CO flow has been detected (Chernin \& Masson 1995; Cernicharo \& Reipurth 1996). Moreover, whereas HH 111 is a strong $\mathrm{H}_{2}$ emitter, with a maximum $\mathrm{H}_{2} 2.12 \mu$ m line flux of $\sim 1.4 \times 10^{-14} \mathrm{erg} \mathrm{s}^{-1} \mathrm{~cm}^{-2}$ from knots L and P, the HH 34 jet shows only a few weak $\mathrm{H}_{2}$ lines, with a maximum flux of $2.5 \times 10^{-15} \mathrm{erg} \mathrm{s}^{-1} \mathrm{~cm}^{-2}$ along the $2.12 \mu \mathrm{m}$ line from knot $\mathrm{C}$. This difference is probably due to the fact that the environment surrounding HH 34 is less dense than the one surrounding $\mathrm{HH}$ 111. In fact, $\mathrm{H}_{2}$ lines are likely to be excited in the bow wings, where the ambient density encountered by the jet is higher than, or comparable to, the jet density and low-velocity shocks $\left(v \sim 20 \mathrm{~km} \mathrm{~s}^{-1}\right)$ are prevalent, preventing $\mathrm{H}_{2}$ dissociation.

\section{Summary and conclusions}

The application of our combined optical/NIR diagnostics to a sample of protostellar jets allows us to define their physical structure, to infer important parameters related to their dynamics such as their mass and linear momentum fluxes, and to investigate the acceleration of associated molecular outflows, as well as the mechanism of dust reprocessing. For the HH 111 and HH 34 jets, which are detected both in the optical and in the near infrared, we obtain the following results:

- In HH 34 the visual extinction, $A_{\mathrm{V}}$, derived from [Fe II] lines varies between $A_{\mathrm{V}} \sim 7.1 \mathrm{mag}$, at a distance of $3^{\prime \prime}$ from the source, to $1.3 \mathrm{mag}$, from $5^{\prime \prime}$ to $30^{\prime \prime}$ from the source. Along $\mathrm{HH} 111$ the visual extinction is $A_{\mathrm{V}} \sim 2$ mag between $15^{\prime \prime}-30^{\prime \prime}$ from the source and negligible in the outer knots.

- In both jets the electron density from the [S II] lines varies between $0.5-3.4 \times 10^{3} \mathrm{~cm}^{-3}$, while in the more compressed region traced by [Fe II] lines we find higher electron density $\left(n_{\mathrm{e}} \sim 1-5 \times 10^{3} \mathrm{~cm}^{-3}\right)$. An even denser layer with electron densities up to $10^{6} \mathrm{~cm}^{-3}$ is traced by [Ca II] lines. Such a density stratification is expected in the post-shocked gas of each unresolved knot.

- A temperature stratification is also found: the temperature derived from optical $\mathrm{S}^{+}, \mathrm{N}^{+}$and $\mathrm{O}$ lines is on average $1.3-1.4 \times 10^{4} \mathrm{~K}$, while in the region of iron emission the temperature is always lower than $10^{4} \mathrm{~K}$.

- In $\mathrm{HH} 111$, which is a strong $\mathrm{H}_{2}$ emitter, we also derive the physical conditions of the molecular component. These lines probably trace the lateral wings of the bow shocks associated with the knot working surfaces, where $\mathrm{H}_{2}$ post-shock temperatures of 2000-3000 K are found.

- The ionisation fraction derived through the BE technique demonstrates that the gas in the jet is only partially ionised $\left(x_{\mathrm{e}} \sim 0.03-0.3\right)$. The derived total density $\left(n_{\mathrm{e}} / x_{\mathrm{e}}\right)$ is about $0.1-3 \times 10^{4} \mathrm{~cm}^{-3}$ in the region of optical emission. If we assume the same ionisation fraction in the more compressed regions where $[\mathrm{Fe} \mathrm{II}]$ and $[\mathrm{Ca} \mathrm{II}]$ lines are excited, total densities up to $10^{7} \mathrm{~cm}^{-3}$ are obtained in the dense layers traced by these lines.
- Estimates of the gas-phase abundance of carbon, calcium and iron are obtained. We find that carbon is not depleted with respect to solar values, while calcium and iron are strongly depleted (the depletion is $\sim 87 \%$ for iron atoms and varies between $70 \%$ and $0 \%$ for calcium). On the other hand, the estimated depletion is lower than the one found in the Orion Cloud by Baldwin et al. (1991) and Esteban et al. (2004) both for Calcium and Iron. This result demonstrates that weak shocks only partially destroy dust in the jet and that some grains still survive. In fact a velocity of $100-400 \mathrm{~km} \mathrm{~s}^{-1}$, like that of a primary bow-shock, is required to completely destroy the dust (Draine 2003).

- Taking into account the filling factor of the gas in the knots, we derive mass flux rates of $\sim 4-5 \times 10^{-8} M_{\odot} \mathrm{yr}^{-1}$. In HH 111 the flux of mass transported by the molecular $\mathrm{H}_{2}$ component is two orders of magnitude lower than the atomic one.

- The estimated flux of linear momentum is on average $\sim 10^{-5} M_{\odot} \mathrm{yr}^{-1} \mathrm{~km} \mathrm{~s}^{-1}$. This is higher than, or comparable to, the flux of momentum measured for the associated $\mathrm{CO}$ molecular outflows, and indicates that jets can drive these molecular outflows.

For the HH 83, HH 73 and $\mathrm{HH} 24 \mathrm{C} / \mathrm{E}$ jets we could only apply optical diagnostics, obtaining the following results:

- The electron densities inferred from the [S II] doublet are very low $\left(n_{\mathrm{e}}<10^{3} \mathrm{~cm}^{-3}\right)$. This explains why these $\mathrm{HH}$ jets are not detected in the NIR. In fact, the transition of [Fe II] lines comes from levels which have a critical density higher than $10^{4} \mathrm{~cm}^{-3}$.

- The ionisation fraction, on the other hand, is always higher in these jets ( $x_{\mathrm{e}}$ up to 0.6), consistent with the fact that for a given shock velocity a higher ionisation is produced when the shock propagates into a low density medium, and with the fact that recombination is slowed down at low electron densities. The latter can also be partially responsible for the higher temperatures in these objects $\left(T_{\mathrm{e}} \sim 1.4-3 \times 10^{4} \mathrm{~K}\right)$, because the collisional cooling process is less efficient at low density. We suggest that the efficiency of the excitation mechanism is the same for all of the jets in our sample, and the differences in the physical conditions of the gas are due to the fact that the $\mathrm{HH} 111$ and $\mathrm{HH} 34$ jets are denser.

- The total density is quite low $\left(n_{\mathrm{H}} \sim 10^{3} \mathrm{~cm}^{-3}\right)$. From these values we infer mass fluxes of $\sim 8 \times 10^{-8} M_{\odot} \mathrm{yr}^{-1}$. Note that these values are upper limits because we are not taking into account the filling factor of the emitting gas for these jets.

- The linear momentum fluxes are of $\sim 1-4 \times$ $10^{-5} M_{\odot} \mathrm{yr}^{-1} \mathrm{~km} \mathrm{~s}^{-1}$.

The above results demonstrate the potential of our combined optical/NIR analysis to study the jet physical structure and represent a good basis to plan future higher spectral-spatial observations with VLT-CRIRES/UVES and AO or interferometric instruments. A higher spectral resolution, in fact, would allow us to analyse the kinematic structure of the jets, while with higher spatial resolution we could extend our analysis to the base of the jet, where it is launched and accelerated, testing the theoretical MHD models.

Acknowledgements. We thank Silvia Medves for letting us use her unpublished reduced spectra to measure [S II] doublet fluxes for the $\mathrm{HH} 111, \mathrm{HH} 83$, $\mathrm{HH} 73$, and $\mathrm{HH} 24 \mathrm{C} / \mathrm{E}$ jets. We are grateful to Francesco Palla and Malcolm Walmsley for useful discussions on elemental abundances and grain destruction processes. We also thank the referee for the many helpful comments to the first version of this paper. This work was partially supported by the European Community's Marie Curie Research and Training Network JETSET 
(Jet Simulations, Experiments and Theory) under contract MRTN-CT-2004005592. J.E. work was partially supported by the Deutsches Zentrum für Luftund Raumfahrt grant 500R0009 and T.P.R.'s research by Science Foundation Ireland grant 04/BRG/P02741.

\section{References}

Asplund, M., Grevesse, N., \& Sauval, A. J. 2005, in Cosmic Abundances as Records of Stellar Evolution and Nucleosynthesis, ASP Conf. Ser., Vol. XXX [arXiv: astro-ph/0410214]

Bacciotti, F. 2002, RMxAC, 13, 8

Bacciotti, F., Chiuderi, C., \& Oliva, E. 1995, A\&A, 296, 185

Bacciotti, F., \& Eislöffel, J. 1999, A\&A, 342, 717 (BE99)

Bacciotti, F., Eislöffel, J., \& Ray, T. P. 1999, A\&A, 350, 917

Bacciotti, F., Ray, T. P., Mundt, R., Eislöffel, J., \& Solf, J. 2002, ApJ, 576, 222

Bacciotti, F., Ray, T. P., Coffey, D., Eislöffel, J., \& Woitas, J. 2004, Ap\&SS, 292, 651

Baldwin, J. A., Ferland, G. J., Martin, P. G., et al. 1991, ApJ, 374, 580

Bally, J., \& Devine, D. 1994, ApJ, 428, L65

Bautista, M. A., Peng, J., \& Pradhan, A. K. 1996, ApJ, 460, 372

Beck-Winchatz, B., Böhm, K. H., \& Noriega-Crespo, A. 1996, AJ, 111, 346

Böhm, K. H., \& Matt, S. 2001, PASP, 113, 158

Böhm, K. H., Brugel, E. W., \& Mannery, E. 1980, ApJ, 235, L137

Brugel, E. W., Böhm, K. H., \& Mannery, E. 1981, ApJS, 47, 117

Cernicharo, J., \& Reipurth, B. 1996, ApJ, 460, 57

Chernin, L. M., \& Masson, C. R. 1995, ApJ, 443, 181

Chidichimo, M. C. 1981, J. Phys. B, 14, 4149

Coffey, D., Bacciotti, F., Woitas, J., Ray, T. P., \& Eislöffel, J. 2004, ApJ, 604, 758

Davis, C. J., Eislöffel, J., \& Smith, M. D. 1996, ApJ, 463, 246

Davis, C. J., Smith, M. D., Eislöffel, J., \& Davies, J. K. 1999, MNRAS, 308, 539

Davis, C. J., Hodapp, K. W., \& Desroches, L. 2001, A\&A, 377, 285

Devine, D., Bally, J., Reipurth, B., \& Heathcote, S. 1997, AJ, 114, 2095D

Draine, B. T. 1989, in Infrared Spectroscopy in Astronomy, ESA-SP290, 93

Draine, B. T. 2003, in The Cold Universe: Saas-Fee Advanced Course 32 [arXiv: astro-ph/0304488]

Eislöffel, J., \& Mundt, R. 1992, A\&A, 263, 292

Eislöffel, J., \& Mundt, R. 1997, AJ, 114, 280

Eislöffel, J., Smith, M. D., \& Davis, C. J. 2000a, A\&A, 359, 1147

Eislöffel, J., Mundt, R., Ray, T. P., \& Rodríguez, L. F. 2000b, Protostars and Planets IV, ed. V. Mannings, A. P. Boss, \& S. S. Russell (Tucson: University of Arizona Press), 815

Esteban, C., Peimbert, M., GarcÃna-Rojas, J., et al. 2004, MNRAS, 355, 229

Flower, D. R., Le Bourlot, J., Pineau des Forêts, G., \& Cabrit, S. 2003, Ap\&SS, 287,183

Giannini, T., McCoey, C., Caratti o Garatti, A., et al. 2004, A\&A, 419, 999

Gredel, R., \& Reipurth, B. 1993, ApJ, 407, L29

Grevesse, N., \& Sauval, A. J. 1998, Space Sci. Rev., 85, 161

Gueth, F., \& Guilloteau, S. 1999, A\&A, 343, 571

Jones, A. P. 2000, J. Geophys. Res., 105, 10257

Hartigan, P. 2003, Ap\&SS, 287, 111

Hartigan, P., Raymond, J., \& Hartmann, L. 1987, ApJ, 316, 323

Hartigan, P., Morse, J. A., \& Raymond, J. 1994, ApJ, 444, 943

Hartigan, P., Morse, J. A., Reipurth, B., Heathcote, S., \& Bally, J. 2001, ApJ, 559, 157

Hartigan, P., Edwards, S., \& Pierson, R. 2004, ApJ, 609, 261

Hillenbrand, L. A., \& White, R. J. 2004, ApJ, 604, 741

Hirth, G. A., Mundt, R., \& Solf, J. 1997, A\&AS, 126, 437

Hollenbach, D., \& McKee, C. F. 1989, ApJ, 342, 306

Hollenbach, D. 1997, IAUS, 182, 181
Königl, A., \& Pudritz, R. E. 2000, Protostar and Planets IV, ed. V. Mannings, A. P. Boss, \& S. S. Russell (Tucson: University of Arizona Press), 759

Landini, M., \& Monsignori Fossi, B. C. 1990, A\&AS, 82, 229

Lane, A. P. 1989, in ESO Workshop on Low Mass Star Formation and Pre-Main Sequence Objects, ed. B. Reipurth (Garching: ESO), 331

Medves, S., Bacciotti, F., \& Eislöffel, J. 2006, in preparation

Mendoza, C. 1983, in Planetary Nebulae, ed. D. R. Flower (Dordrecht: Reidel), IAU Symp., 103, 143

Moneti, A., \& Reipurth, B. 1995, A\&A, 301, 721

Morse, J. A., Hartigan, P., Cecil, G., Raymond, J., \& Heathcote, S. 1992, ApJ, 399,231

Morse, J. A., Heathcote, S., Hartigan, P., \& Cecil, G. 1993, AJ, 106, 1139

Mundt, R., Ray, T. P., \& Raga, A. C. 1991, A\&A, 252, 740

Mouri, H., \& Taniguchi, Y. 2000, ApJ, 534, L63

Nisini, B., Caratti o Garatti, A., Giannini, T., \& Lorenzetti, D. 2002, A\&A, 393, 1035

Nisini, B., Antoniucci, S., Giannini, T., \& Lorenzetti, D. 2005a, A\&A, 429, 543

Nisini, B., Bacciotti, F., Giannini, T., et al. 2005b, A\&A, 441, 159

Noriega-Crespo, A., Garnavich, P. M., \& Raga, A. C. 1993, AJ, 106, 1133

Nussbaumer, H., \& Storey, P. J. 1988, A\&A, 193, 327

Oliva, E., Marconi, A., Maiolino, R., et al. 2001, A\&A, 369, L50

Osterbrock, D. E. 1994, Astrophysics of Gaseous Nebulae and Active Galactic Nuclei (Mill Valley: University Science Book)

Pesenti, N., Dougados, C., Cabrit, S., et al. 2003, A\&A, 410, 155

Peimbert, M., Torres-Peimbert, S., \& Dufour, R. J. 1993, ApJ, 418, 760

Quinet, P., Le Dourneuf, M., \& Zeippen, C. J. 1996, A\&AS, 120, 361

Raga, A. C., \& Böhm, K. H. 1986, ApJ, 308, 829

Raga, A. C., \& Kofman, L. 1992, ApJ, 386, 222

Raga, A. C., Noriega-Crespo, A., Reipurth, B., et al. 2002, ApJ, 565, L29

Ray, T. P., \& Bacciotti, F. 2003, RMxAC, 15, 106

Ray, T. P., Mundt, R., Dyson, J. E., Falle, S. A. E. G., \& Raga, A. C. 1996, ApJ, 468, L103

Reipurth, B. 1981, A\&A, 44, 379

Reipurth, B. 1989, A\&A, 220, 249

Reipurth, B., \& Olberg, M. 1991, A\&A, 246, 535

Reipurth, B., \& Bally, J. 2001, ARA\&A, 39, 403

Reipurth, B., \& Graham, J. A. 1988, A\&A, 202, 219

Reipurth, B., Bally, J., Graham, J. A., Lane, A. P., \& Zealey, W. J. 1986, A\&A, 164,51

Reipurth, B., Hartigan, P., Heathcote, S., Morse, J. A., \& Bally, J. 1997, AJ, 114, 757

Reipurth, B., Yu, K. C., Heathcote, S., Bally, J., \& Rodriguez, L. F. 2000, AJ, 120,1449

Reipurth, B., Heathcote, S., Morse, J., Hartigan, P., \& Bally, J. 2002, AJ, 123, 362

Rieke, G. H., \& Lebofsky, M. J. 1985, ApJ, 288, 618

Rolph, C. D., Scarrott, S. M., \& Wolstencroft, R. D. 1990, MNRAS, 242, 109

Rubin, R. H., Simpson, J. P., Haas, M. R., \& Erickson, E. F. 1991, ApJ, 374, 564

Shang, H., Glassgold, A. E., Shu, F. H., \& Lizano, S. 2002, ApJ, 564, 853

Shu, F. H., Najita, J. R., Shang, H., \& Li, Z.-Y. 2000, Protostar and Planets IV, ed. V. Mannings, A. P. Boss, \& S. S. Russell (Tucson: University of Arizona Press), 789

Smith, N., \& Hartigan, P. 2006, ApJ, 638, 1045

Solf, J. 1987, A\&A, 184, 322

Stancil, P. C., Havener, C. C., Krstic, P. S., et al. 1998, ApJ, 502, 1006

Stanke, T., McCaughrean, M. J., \& Zinnecker, H. 1998, A\&A, 332, 307

Stapelfeldt, K. R., Scoville, N. Z., Beichman, C. A., Hester, J. J., \& Gautier III, T. N. 1991, ApJ, 371, 226

Wilson, T. L., \& Rood, R. T. 1994, ARA\&A, 32, 191

Woitas, J., Bacciotti, F., Ray, T. P., et al. 2005, A\&A, 432, 149

Zealey, W. J., Mundt, R., Ray, T. P., et al. 1989, PASAu, 8, 62 